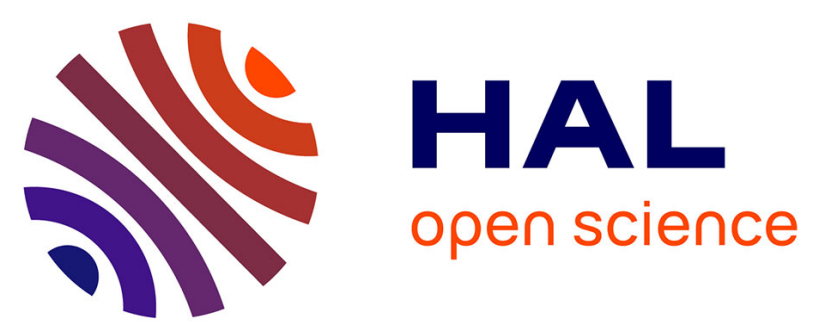

\title{
On the identification of the blood vessel confounding effect in intravoxel incoherent motion (IVIM) Diffusion-Weighted (DW)-MRI in liver: An efficient sparsity based algorithm
}

Jie Liu, Giulio Gambarota, Huazhong Shu, Longyu Jiang, Benjamin Leporq, Olivier Beuf, Ahmad Karfoul

\section{To cite this version:}

Jie Liu, Giulio Gambarota, Huazhong Shu, Longyu Jiang, Benjamin Leporq, et al.. On the identification of the blood vessel confounding effect in intravoxel incoherent motion (IVIM) DiffusionWeighted (DW)-MRI in liver: An efficient sparsity based algorithm. Medical Image Analysis, 2020, 61, pp.101637. 10.1016/j.media.2020.101637 . hal-02441136

\author{
HAL Id: hal-02441136 \\ https://hal.science/hal-02441136
}

Submitted on 21 Feb 2020

HAL is a multi-disciplinary open access archive for the deposit and dissemination of scientific research documents, whether they are published or not. The documents may come from teaching and research institutions in France or abroad, or from public or private research centers.
L'archive ouverte pluridisciplinaire HAL, est destinée au dépôt et à la diffusion de documents scientifiques de niveau recherche, publiés ou non, émanant des établissements d'enseignement et de recherche français ou étrangers, des laboratoires publics ou privés. 


\section{Highlights}

- All-voxel IVIM-MRI model for simultaneous processing of all voxel in a given ROI.

- Ability to incorporate several priors on the model parameters

- High detection of blood vessels in the ROI 


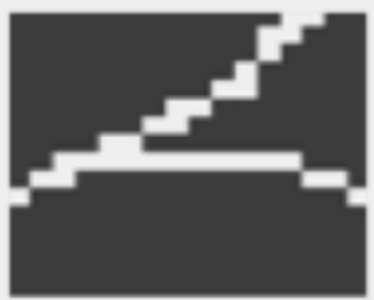

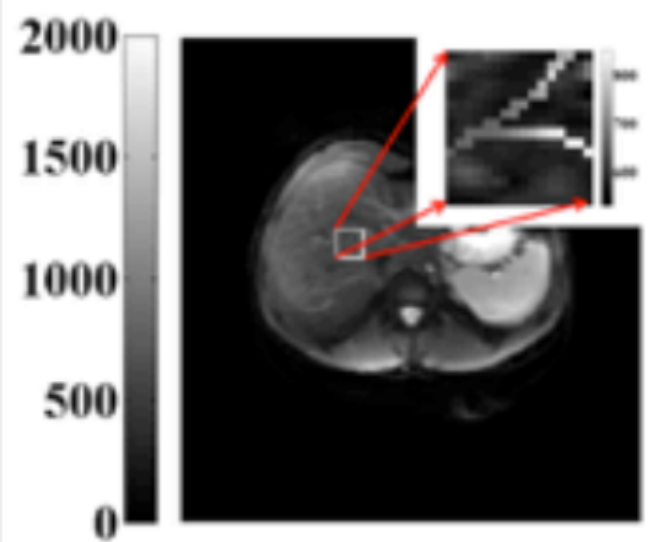

W-MR image with difficult visual identification of blood vessels
Constrained all-voxel IVIM-MRI model
Blood vessels detection r.

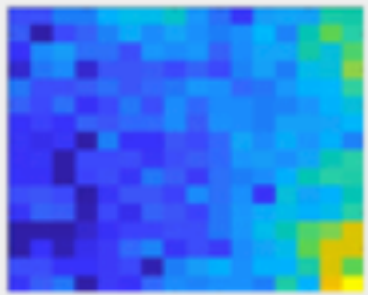

Parametric map of tissue's apparent diffusion coefficient

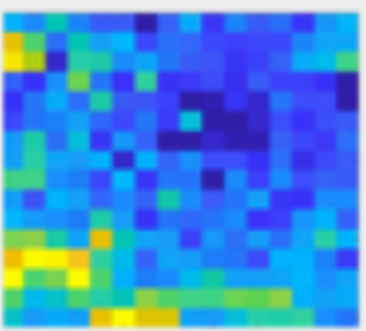

Parametric map of tissue's perfusion fraction 


\title{
On the identification of the blood vessel confounding effect in intravoxel incoherent motion (IVIM) Diffusion-Weighted (DW)-MRI in liver: An efficient sparsity based algorithm
}

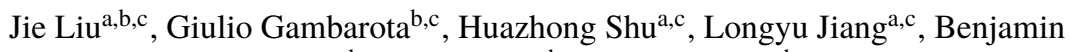 \\ Leporq $^{\mathrm{d}}$, Olivier Beuf ${ }^{\mathrm{d}}$, Ahmad Karfoul $^{\mathrm{b}, \mathrm{c}, *}$ \\ ${ }^{a}$ Laboratory of Image Science and Technology (LIST), School of Computer Science and Engineering, \\ Southeast University, Nanjing, 210096, China \\ ${ }^{b}$ Univ Rennes, INSERM, LTSI-UMR 1099, F-35000, Rennes France \\ ${ }^{c}$ Univ Rennes, Southeast University, INSERM, Centre de Recherche en Information Biomédicale \\ sino-français (CRIBs)- LIA, F-35000, Rennes, France \\ ${ }^{d}$ Univ Lyon, INSA-Lyon, Université Claude Bernard Lyon 1, UJM-Saint Etienne, CNRS, Inserm, CREATIS \\ UMR 5220, U1206, F-69621 Lyon, France
}

\begin{abstract}
IntraVoxel Incoherent Motion (IVIM) Diffusion-Weighted Magnetic Resonance Imaging (DW-MRI) is of great interest for evaluating tissue diffusion and perfusion and producing parametric maps in clinical applications for liver pathologies. However, the presence of macroscopic blood vessels (not capillaries) in a given Region of Interest (ROI) results in a confounding effect that bias the quantification of tissue perfusion. Therefore, it is necessary to identify those voxels affected by blood vessels. In this paper, an efficient algorithm for an automatic identification of blood vessels in a given ROI is proposed. It relies on the sparsity of the spatial distribution of blood vessels. This sparsity prior can be easily incorporated using the all-voxel IVIM-MRI model introduced in this paper. In addition to the identification of blood vessels, the proposed algorithm provides a quantification of blood vessels, tissue diffusion and tissue perfusion of all voxels in a given ROI, in one single step. Besides, two strategies are proposed in this paper to deal with the nonnegativity of the model parameters. The efficiency of the proposed algorithm compared to the Non-Negative Least Square (NNLS)-based method, recently introduced to deal with the confounding blood vessel effect in the
\end{abstract}

\footnotetext{
${ }^{*}$ Corresponding author. Tel: +33-2-23233124, Fax: +33-2-23236917, Laboratoire Traitement du Signal et de l'Image (LTSI), Bât.22, Campus de Beaulieu, Univ Rennes 1, 35042 Rennes, France

Email address: ahmad.karfoul@univ-rennes1.fr (Ahmad Karfoul)
} 
IVIM-MRI model, is confirmed using both realistic and real DW-MR images.

Keywords: IVIM-MRI, Diffusion-Weighted MRI, sparsity, proximal optimization.

\section{Introduction}

IntraVoxel Incoherent Motion (IVIM) imaging is a method to quantitatively assess the microscopic translational motions of water molecules that could contribute to the signal acquired with diffusion-weighted magnetic resonance imaging (DW-MRI) 5 (Le Bihan et al., 1986, 1988; Le Bihan, 2008). The IVIM-MRI model comprises two distinct contributions: the molecular diffusion of water in tissue (also referred to as "true diffusion") and the microcirculation of blood in the capillaries (perfusion, also referred to as "pseudo diffusion"). Thus, the IVIM-MRI method allows for measurements of both the apparent diffusion coefficient (ADC) and the tissue perfusion, in a

10 single experiment. The ADC can be used to probe the tissue microstructure and represents a diagnostic biomarker for a number of diseases. The tissue perfusion is another important physiological parameter that is sensitive to tissue disease status. It should be noted that, contrary to other perfusion techniques, the IVIM-MRI provides an index of perfusion without the intravenous administration of a contrast agent (e.g., Gd-DTPA). Despite the limited clinical application of IVIM perfusion maps (due to their noisiness), many IVIM-MRI studies have been performed recently on liver to investigate pathologies such as nonalcoholic fatty liver disease (NAFLD) and steatohepatitis (NASH), which are nowadays reaching epidemic levels worldwide (Loomba and Sanyal, 2013; Andreou et al., 2013; Dyvorne et al., 2013; Hayashi et al., 2013; Pasquinelli et al., 2011; Guiu et al., 2012; Leporq et al., 2015).

The IVIM-MRI model is bi-exponential since the diffusion signal at each voxel is modeled as a linear combination of two exponential decays related to the ADC and the tissue perfusion (more details are given in section 2). Recently, two studies have highlighted a possible presence of a third contribution to the diffusion signal, which 25 could be found in voxels affected by larger blood vessels (not capillaries) (Cercueil et al., 2015; Gambarota et al., 2017). This contribution can be modeled as a third diffusion decay component leading to a tri-exponential IVIM-MRI model. It is noteworthy that 
this third component is not necessarily present in all voxels but only in those potentially affected by large blood vessels (i.e., partial volume). A trivial way to quantify tissue diffusion and perfusion of the Region of Interest (ROI) affected by the presence of blood vessels is first to visually discard those affected voxels and second to estimate the ADC and the tissue perfusion by fitting a classical bi-exponential IVIM-MRI model to the remaining ones. However, such approach requires a sufficiently high contrast level in the ROI under consideration. In general, due to the limited signal contrast and 35 spatial resolution, the visual identification of affected voxels and hence the estimation of a potential third diffusion component becomes a non-trivial task. In such situation, authors in (Gambarota et al., 2017) proposed to investigate, for each voxel in the ROI, the presence of large blood vessels (i.e., third diffusion component) using the Non-Negative Least Square (NNLS) algorithm (Lawson and Hanson, 1995). For each voxel labeled 40 as affected by a blood vessel, the confounding effect of the blood vessel was "removed" via a heuristic strategy (e.g., by discarding the data point related to the contribution of blood vessels to the signal intensity) (Gambarota et al., 2017). After performing this pre-processing step, a bi-exponential fitting was used to estimate both the ADC and the tissue perfusion. Besides, authors in (Cercueil et al., 2015) adopted a tri-exponential 45 IVIM-MRI model fit using nonlinear regression methods. In this study, the estimation of the third component was not performed on a voxel-by-voxel basis but using the ROI's mean intensity (i.e. the average intensity over all the voxels in the ROI). Therefore, no parametric maps could be generated with such a strategy neither for blood vessels nor for $\mathrm{ADC}$ and tissue perfusion. Since these parametric maps are highly valuable from ${ }_{50}$ a diagnostic point of view, the approach by (Cercueil et al., 2015) is not considered in the current paper.

Given the great clinical interest in the IVIM-MRI method for quantifying tissue diffusion and perfusion, especially for ROIs where the identification of blood vessels is not evident, a new algorithm for automatic identification of the potential presence of 55 blood vessels in a given ROI is proposed in this paper. In addition to this identification, which is the main goal of this algorithm, the contribution of (i) blood vessels, (ii) the $\mathrm{ADC}$ and (iii) the tissue perfusion to the acquired signals, is quantified for all voxels in the ROI, in one single step. This is accomplished with the all-voxel IVIM-MRI 
model introduced in this paper. The proposed algorithm does not require any manual 60 operations, such as image contrast enhancement commonly used in such context, which are operator-dependent and prone to errors. The proposed algorithm takes into account (i) the assumption of a sparse representation of the spatial distribution of large blood vessels in the considered ROI leading to the Sparsity-based All-Voxel Tri-Exponential IVIM (SAVTE-IVIM) algorithm; and (ii) the non-negativity constraint that is inherent to the IVIM-MRI model. To deal with this non-negativity constraints, two possible strategies are proposed in this paper: (i) a rough strategy leading to the SAVTEIVIM $_{\mathrm{R}}$ method; and (ii) an embedded strategy leading to the SAVTE-IVIM $\mathrm{E}_{\mathrm{E}}$ one. The blood vessel identification and parameter quantification are computed following the spirit of the Alternating Direction Method of Multipliers (ADMM) (Boyd et al., 70 2011). The performance of the proposed SAVTE-IVIM algorithm (in its two variants

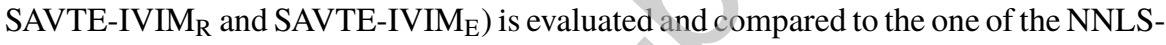
based method (Gambarota et al., 2017) in terms of identifying blood vessels and quantifying tissue diffusion and perfusion. A statistical analysis of obtained results in both identifying blood vessels and quantifying tissue diffusion and perfusion, is also 75 performed. Both realistic simulated and real DW-MRI images of liver acquired from six healthy volunteers are used for the aforementioned numerical analysis. This paper is an extended version of our preliminary work reported in (Liu et al., 2017, 2018).

\section{Method}

\subsection{Towards an all-voxel tri-exponential IVIM-MRI model}

Assume, for a given volunteer, that a set of $M$ DW-MR images acquired using $M$ different diffusion-sensitizing gradient strengths (denoted here by $b_{m}, m \in\{1, \cdots, M\}$ ) is available. Then, for a given voxel in an ROI of size $\left(N_{1} \times N_{2}\right)$ taken in the $m$-th DW-MR image, the conventional IVIM-MRI model is given by (Le Bihan et al., 1986):

$$
s^{\left(n_{1}, n_{2}\right)}\left(b_{m}\right)=\sum_{\ell=1}^{L=2} a_{\ell}^{\left(n_{1}, n_{2}\right)} e^{-b_{m} d_{\ell}^{\left(n_{1}, n_{2}\right)}}+\epsilon^{\left(n_{1}, n_{2}\right)}\left(b_{m}\right)
$$

85 where $s^{\left(n_{1}, n_{2}\right)}$ denotes the acquired signal intensity of the $\left(n_{1}, n_{2}\right)$-th voxel, $1 \leq n_{1} \leq$ $N_{1}, 1 \leq n_{2} \leq N_{2}$, and $\epsilon^{\left(n_{1}, n_{2}\right)}$ is an additive Rician noise. Coefficients $d_{1}^{\left(n_{1}, n_{2}\right)}$ and 
$d_{2}^{\left(n_{1}, n_{2}\right)}$ with $d_{1}^{\left(n_{1}, n_{2}\right)}<d_{2}^{\left(n_{1}, n_{2}\right)}$ stand respectively for the ADC (characterizing the slow exponential decay, around $10^{-3} \mathrm{~mm}^{2} / \mathrm{sec}$ ) and the $D^{*}$ (the pseudo-diffusion coefficient, characterizing the fast exponential decay, around $10^{-1} \mathrm{~mm}^{2} / \mathrm{sec}$, which originates from 90 the blood perfusion); $a_{1}^{\left(n_{1}, n_{2}\right)}$, and $a_{2}^{\left(n_{1}, n_{2}\right)}$ are their corresponding amplitudes. According to equation (1), the IVIM-MRI model provides a means to simultaneously assess the ADC and the perfusion fraction (PF) which is defined as a normalized amplitude of the fast diffusion component (Gambarota et al., 2017) such that for the $\left(n_{1}, n_{2}\right)$-th voxel we have: $\mathrm{PF}^{\left(n_{1}, n_{2}\right)}=\frac{a_{2}^{\left(n_{1}, n_{2}\right)}}{\sum_{\ell=1}^{L} a_{\ell}^{\left(n_{1}, n_{2}\right)}}$. However, this conventional IVIM-MRI model does 95 not take into account the potential contribution of a large blood vessel in the considered $\left(n_{1}, n_{2}\right)$-th voxel $\left(1 \leq n_{1} \leq N_{1}, 1 \leq n_{2} \leq N_{2}\right)$ to the acquired signal. Authors in (Gambarota et al., 2017; Cercueil et al., 2015) investigated the presence of large blood vessels and suggested to consider tri-exponential IVIM-MRI model (e.g., $L=3$ in equation (1)) as a mean to capture such blood vessel effect. Consequently, in addition to the slow (i.e., $\left.d_{1}^{\left(n_{1}, n_{2}\right)}(\mathrm{ADC})\right)$ and the fast (i.e., $\left.d_{2}^{\left(n_{1}, n_{2}\right)}\left(\mathrm{D}^{*}\right)\right)$ exponential decays, the tri-exponential IVIM-MRI model (equation (1)) comprises a third very fast exponential decay (i.e., $d_{3}^{\left(n_{1}, n_{2}\right)}>0.2 \mathrm{~mm}^{2} / \mathrm{sec}$ ) with related amplitude $a_{3}^{\left(n_{1}, n_{2}\right)}$. Despite the efficiency, to some extent, of methods in (Gambarota et al., 2017; Cercueil et al., 2015), none of them are able to consider possible prior information regarding the spatial distribution of the model parameters. Specifically, the use of prior information might considerably improve the identification of blood vessels in the considered ROI. This limitation of the previously proposed methods (Gambarota et al., 2017; Cercueil et al., 2015 ) is due to the fact that the employed tri-exponential IVIM-MRI model (e.g., $L=3$ in equation (1)) is, conventionally, a single voxel-wise model. To cope with this limitation, the latter model is extended, as shown hereafter, to an all-voxel tri-exponential IVIM-MRI one. With this extended version being considered, not only spatial prior regarding the model parameters can be employed, but also a simultaneous processing of all voxels in the considered ROI is henceforth possible. For convenience purposes, a vectorized version of any defined ROI in the DW-MR image will be adopted in the sequel. Such vectorization is performed using the vec operator which maps a matrix $\boldsymbol{T}(I \times J)$ to an $I J$-th dimensional vector whose $i+(j-1) I$-th component stands for the $(i, j)$-th entry of $\boldsymbol{T}$. Consequently, its inverse function, denoted by unvec, is defined 
such that $\operatorname{unvec}(\operatorname{vec}(\boldsymbol{T}))=\boldsymbol{T}$. Accordingly, based on equation (1) (for $L=3$ ) and for a given ROI in the $m$-th $(m \in\{1, \cdots, M\})$ DW-MRI image, the all-voxel tri-exponential

IVIM-MRI model can be written as:

$$
\boldsymbol{s}\left(b_{m}\right)=\sum_{\ell=1}^{L=3} \boldsymbol{a}_{\ell} \boxminus e^{-b_{m} \boldsymbol{d}_{\ell}}+\boldsymbol{\epsilon}\left(b_{m}\right)
$$

where $\boldsymbol{a}_{\ell}=\operatorname{vec}\left(\boldsymbol{A}_{\ell}\right)$ and $\boldsymbol{d}_{\ell}=\operatorname{vec}\left(\boldsymbol{D}_{\ell}\right), \forall \ell \in\{1,2,3\}$ with matrices $\boldsymbol{A}_{l}, \boldsymbol{D}_{l}$ of size $\left(N_{1} \times N_{2}\right)$ denoting respectively the spatial distribution of $a_{\ell}$ and $d_{\ell}$ in the considered ROI, $\square$ stands for the Hadamard product (i.e., element-wise matrix product) and $\boldsymbol{\epsilon}\left(b_{m}\right)$ denotes the $N_{1} N_{2}$-th dimensional vector of MRI noise related to the $m$-th image. Since spatial distributions $\boldsymbol{A}_{\ell}, \boldsymbol{D}_{\ell}, \ell \in\{1,2,3\}$ are invariant through the $M$ DW-MR images (Le Bihan et al., 1986), the all-voxel tri-exponential IVIM-MRI model defined over the $M$ DW-MR images can then be written as:

$$
\boldsymbol{s}=\sum_{\ell=1}^{L=3}\left(\mathbf{1}_{M} \otimes \boldsymbol{a}_{\ell}\right) \bullet e^{-\boldsymbol{b} \otimes \boldsymbol{d}_{\ell}}+\boldsymbol{\epsilon}
$$

where $s$ is a $N M$-dimensional vector gathering the acquired signals of the $N=N_{1} N_{2}$ voxels of the considered ROI over the $M$ DW-MR images. The noise vector $\epsilon$, is defined such that $\boldsymbol{\epsilon}=\left[\boldsymbol{\epsilon}\left(b_{1}\right)^{\top}, \boldsymbol{\epsilon}\left(b_{2}\right)^{\top}, \cdots, \boldsymbol{\epsilon}\left(b_{M}\right)^{\top}\right]^{\top}, \mathbf{1}_{M}$ is an $M$-dimensional vector of ones, $\boldsymbol{b}=\left[b_{1}, b_{2}, \cdots, b_{M}\right]^{\top}$ where ${ }^{\top}$ is the transpose operator and $\otimes$ stands for the Kronecker product.

\subsection{The proposed SAVTE-IVIM algorithm}

A reliable clinical diagnosis based on a given ROI in the liver, when the presence of blood vessels is questionable, requires an efficient way of detecting blood vessels. As clinical experts are generally interested in investigating the tissue status, delineating a ROI that is, to a large extent, free from the confounding blood vessel effect is mandatory. In this sense, a chosen ROI is considered as informative in terms of both $\mathrm{ADC}$ and $\mathrm{PF}$ when the spatial distribution of blood vessels, in this ROI, is sparse. More precisely, the spatial distribution, $\boldsymbol{A}_{3}$, of $a_{3}$ is a sparse matrix. As the spatial distribution of blood vessels in the ROI reflects its vascularization, the sparsity pattern of $\boldsymbol{A}_{3}$ is invariant through the $M$ DW-MR images. Therefore, identifying blood vessels in the considered 
ROI can be performed by solving the following model identification problem:

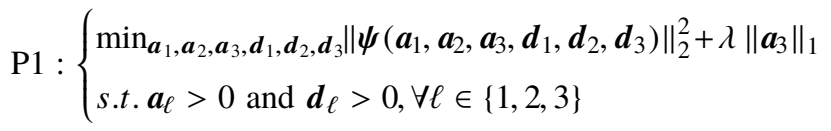

where $\psi\left(\boldsymbol{a}_{1}, \boldsymbol{a}_{2}, \boldsymbol{a}_{3}, \boldsymbol{d}_{1}, \boldsymbol{d}_{2}, \boldsymbol{d}_{3}\right)=\boldsymbol{s}-\sum_{\ell=1}^{L=3}\left(\mathbf{1}_{M} \otimes \mathbf{a}_{\ell}\right) \varpi e^{-\boldsymbol{b} \otimes \boldsymbol{d}_{\ell}}$ denotes the model fidelity,

$\|.\|_{2}^{2},\|.\|_{1}$ stand, respectively, for $\ell_{2}$-norm and $\ell_{1}$-norm and $\lambda$ stands for a penalty parameter. For the sake of readability, arguments in the function $\psi\left(\boldsymbol{a}_{1}, \boldsymbol{a}_{2}, \boldsymbol{a}_{3}, \boldsymbol{d}_{1}, \boldsymbol{d}_{2}, \boldsymbol{d}_{3}\right)$ will be omitted in the sequel. Note that a LASSO (Least Absolute Shrinkage and Selection Operator) penalty term (i.e., $\ell_{1}$-norm) is used in P1 (4) to describe the sparsity of the spatial distribution $\boldsymbol{A}_{3}$ of $a_{3}$. The above constrained minimization problem, P1 (4), can be solved following the spirit of the well-known ADMM method which is based on the augmented Lagrangian technique (Boyd et al., 2011). Regarding the non-negativity constraints, rough and embedded strategies are proposed in this paper leading, as mentioned previously, to the SAVTE-IVIM $\mathrm{R}_{\mathrm{R}}$ and the SAVTE-IVIM algorithms, respectively.

\subsubsection{The SAVTE-IVIM $M_{\mathrm{R}}$ algorithm}

The P1 problem (4) is solved here using the ADMM method (Boyd et al., 2011) which introduces the latent variable $z$ and replaces the P1 problem (4) by:

$$
\mathrm{P} 2:\left\{\begin{array}{l}
\min _{\boldsymbol{a}_{1}, \boldsymbol{a}_{2}, \boldsymbol{a}_{3}, \boldsymbol{d}_{1}, \boldsymbol{d}_{2}, \boldsymbol{d}_{3}, z} \quad\|\boldsymbol{\psi}\|_{2}^{2}+\lambda\|z\|_{1} \\
\text { s.t. } \quad z=\boldsymbol{a}_{3}, \boldsymbol{a}_{\ell}>0, \boldsymbol{d}_{\ell}>0, \forall \ell \in\{1,2,3\}
\end{array}\right.
$$

A typical scheme to solve P2 (5) using the ADMM method is based on the minimization of its associated augmented Lagrangian function, denoted here by $\mathcal{L}$. This leads to rewrite P2 (5) as follows:

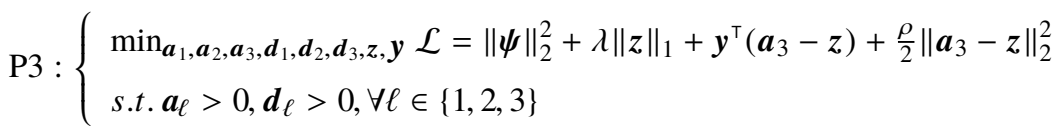

where the $N$-dimensional vector $\boldsymbol{y}$ stands for the Lagrangian multiplier and $\rho>0$ denotes a penalty parameter. The estimation of $\boldsymbol{a}_{\ell}, \forall \ell \in\{1,2,3\}$, can be then performed in a least square sense by computing the stationary points of $\mathcal{L}$ in $\boldsymbol{a}_{\ell}, \forall \ell \in\{1,2,3\}$. 
Regarding the estimation of $\boldsymbol{d}_{1}, \boldsymbol{d}_{2}$ and $\boldsymbol{d}_{3}$, it is performed using the LevenbergMarquardt (LM) algorithm (Nocedal and Wright, 2006). Note that instead of using the LM algorithm, other alternative non-linear methods such as the Gauss-Newton (GN) (Boyd and Vandenberghe, 2004) can be employed instead, as shown in our preliminary work (Liu et al., 2017). However, the LM algorithm yielded a higher performance in terms of the estimation quality compared to the GN algorithm (Liu et al., 2017). Therefore, and for the sake of readability, only the LM algorithm is considered in this paper. As far as the estimation of the latent variable $z$ and the Lagrange multiplier $\boldsymbol{y}$ is concerned, a proximal operator (see (Komodakis and Pesquet, 2015) and the references therein) is used to estimate the former while the dual ascent scheme (Boyd et al., 2011) is used to estimate the latter. Mathematical derivations of all update rules employed to solve the P3 problem (6), in the framework of the ADMM method, are given in Appendix A. At each iteration of the SAVTE-IVIM $\mathrm{R}_{\mathrm{R}}$ algorithm, variables are updated in an alternative way. Indeed, each variable is estimated while keeping the other variables to their last estimates. The algorithm stops when the model error exhibits, between two successive iterations, a value that is smaller than or equal to a predefined threshold or when a maximum number of iterations is reached. As for the non-negativity constraints in $\mathrm{P} 2 / \mathrm{P} 3$, a rough strategy is used to deal with it. More precisely, potential negative components in $\boldsymbol{a}_{1}, \boldsymbol{a}_{2}$ and $\boldsymbol{a}_{3}$ are set to a value in the neighborhood of zero (e.g., $10^{-5}$ ). The choice of the latter threshold value is justified since no physiological prior information regarding the value of $\boldsymbol{a}_{\ell}, \forall \ell \in\{1,2,3\}$ is to be considered. Regarding negative components of $\boldsymbol{d}_{1}$ and $\boldsymbol{d}_{2}$ encountered during iterations, they are projected back to their respective physiological ranges $d_{1}^{(n)}<0.01 d_{2}^{(n)} \in[0.01,0.2]$ and $d_{3}^{(n)}>0.2$, where $d_{\ell}^{(n)}, \ell \in\{1,2,3\}$ stands for the $n$-th component of the $N$-dimensional vector $\boldsymbol{d}_{\ell}$. Indeed, potential negative values of $d_{1}^{(n)}$ and $d_{2}^{(n)}$ are set to $10^{-5}, 0.01$, respectively. Also, values of $d_{1}$ that are greater than 0.01 are set to 0.01 while those of $d_{2}^{(n)}\left(d_{3}^{(n)}\right)$ that are greater(smaller) than 0.2 are set to 0.2 . A pseudo code summarizing the main steps of the proposed SAVTE-IVIM $\mathrm{R}$ algorithm is given in Algorithm 1 hereafter. 


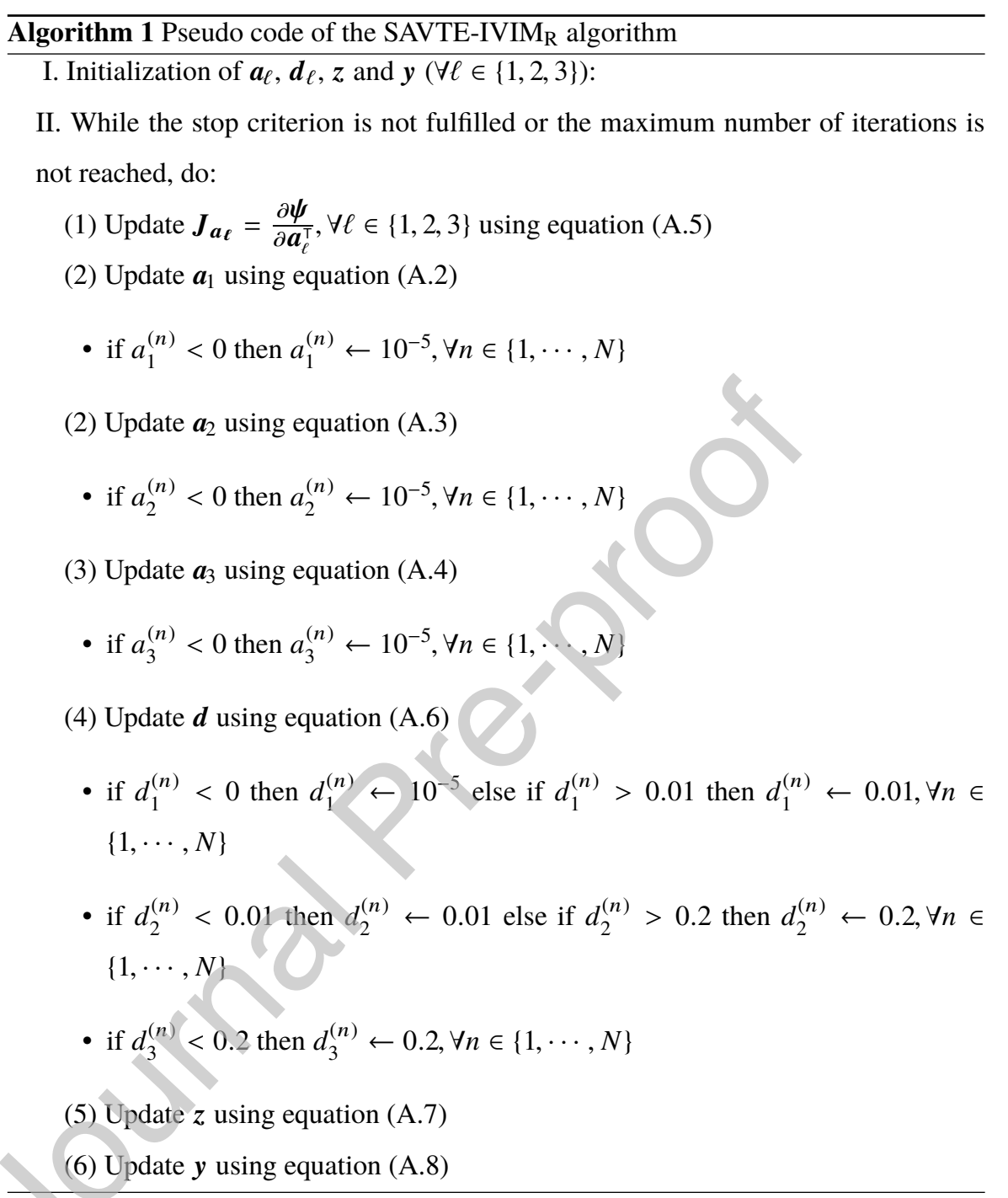

\subsubsection{The SAVTE-IVIM $\mathrm{E}_{\mathrm{E}}$ algorithm}

The SAVTE-IVIM $\mathrm{E}$ algorithm deals with non-negativity constraints in P1 (4) by resorting to a change of variable into square such that: $\boldsymbol{a}_{\ell}=\tilde{\boldsymbol{a}_{\ell}} \boxminus \tilde{\boldsymbol{a}_{\ell}}=\tilde{\boldsymbol{a}}_{\ell}^{\square 2}, \boldsymbol{d}_{\ell}=$ $\tilde{\boldsymbol{d}} \ell \square \tilde{\boldsymbol{d}} \ell=\tilde{\boldsymbol{d}}_{\ell}{ }^{\square 2}$ with $\tilde{\boldsymbol{a}}_{\ell}, \tilde{\boldsymbol{d}}_{\ell} \in \mathbb{R}^{*}, \forall \ell \in\{1,2,3\}$ (see Coloigner et al. (2014) and the references therein). As the sparsity patterns are invariant w.r.t. the Hadamard product (e.g $\boldsymbol{a}_{3}$ and $\tilde{\boldsymbol{a}_{3}}$ have the same sparsity pattern), P1 (4) can then be reformulated as 
follows:

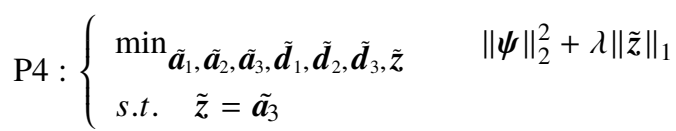

where $\psi=s-\sum_{\ell=1}^{L=3}\left(\mathbf{1}_{M} \otimes \tilde{\boldsymbol{a}}_{\ell}{ }^{\square 2}\right) \varpi e^{-\boldsymbol{b} \otimes \tilde{\boldsymbol{d}}_{\ell}{ }^{\boxminus 2}}$. Similarly to the SAVTE-IVIM $\mathrm{R}$ approach, the ADMM method is employed to solve the above optimization problem. To do so, $\mathrm{P} 4$ (7) is reformulated as follows:

$$
\mathrm{P} 5: \min _{\tilde{\boldsymbol{a}}_{1}, \tilde{\boldsymbol{a}}_{2}, \tilde{\boldsymbol{a}}_{3}, \tilde{\boldsymbol{d}}_{1}, \tilde{\boldsymbol{d}}_{2}, \tilde{\boldsymbol{d}}_{3}, \tilde{\boldsymbol{z}}, \tilde{\boldsymbol{y}}} \mathcal{L}_{1}=\|\boldsymbol{\psi}\|_{2}^{2}+\lambda\|\tilde{\boldsymbol{z}}\|_{1}+\tilde{\boldsymbol{y}}^{\top}\left(\tilde{\boldsymbol{a}_{3}}-\tilde{z}\right)+\frac{\rho}{2}\left\|\tilde{\boldsymbol{a}_{3}}-\tilde{\boldsymbol{z}}\right\|_{2}^{2}
$$

where $\mathcal{L}_{1}$ is its associated Lagrangian function. Parameters $\boldsymbol{a}_{\ell}, \forall \ell \in\{1,2,3\}$, are estimated also by computing the stationary points of $\mathcal{L}_{1}$ in $\boldsymbol{a}_{\ell}, \forall \ell \in\{1,2,3\}$. Regarding the estimation of $\boldsymbol{d}_{\ell}, \forall \ell \in\{1,2,3\}$, the LM method is also employed. Like the SAVTEIVIM $_{R}$, parameter estimation is also performed in an alternative way. Indeed, at each iteration, each parameter is updated while keeping the other ones fixed to their last estimates. The algorithm stops when the model error exhibits, between two successive iterations, a value that is smaller than or equal to a predefined threshold or when a maximum number of iterations is reached. A pseudo code summarizing the different steps in the proposed SAVTE-IVIM $\mathrm{E}_{\mathrm{E}}$ algorithm is given in Algorithm 2 below.

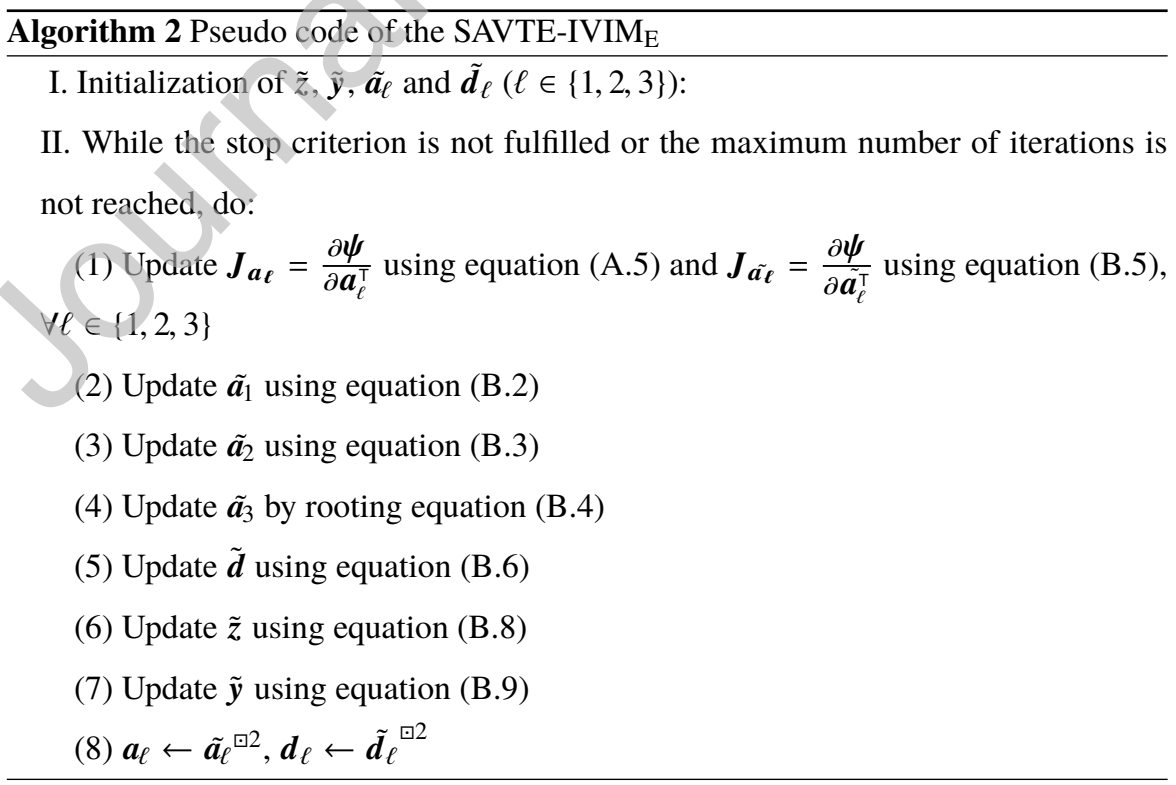


Mathematical derivations of all update rules figured in Algorithm 2 are given in Appendix B.

The numerical complexity of the proposed SAVTE-IVIM algorithm in its two variants, the SAVTE-IVIM $\mathrm{R}_{\mathrm{R}}$ and SAVTE-IVIM $\mathrm{E}$, is expressed in numerical flop. A flop is defined as a multiplication followed by addition. However, since in practice more multiplications than additions are encountered, only multiplications are taken into account here. Thus, the numerical complexity per iteration of the SAVTE-IVIM $\mathrm{I}_{\mathrm{R}}$ algorithm is equal to $(11 M+30) N^{3}+(6 M+9) N^{2}+(39 M+5) N+5$ flops while the one of the SAVTEIVIM $_{\mathrm{E}}$ is equal to $(22 M+29) N^{3}+(6 M+9) N^{2}+(39 M+12) N+5$ flops, where as mentioned previously, $N$ denotes the number of voxels in the considered ROI, and $M$ stands for the number of b-values.

\section{Simulations and results}

This section is devoted to evaluate the behavior of the proposed SAVTE-IVIM ${ }_{225}$ algorithm in its two variants, the SAVTE-IVIM $\mathrm{R}_{\mathrm{R}}$ and the SAVTE-IVIM $\mathrm{S}_{\mathrm{E}}$, compared to the NNLS-based method (Gambarota et al., 2017) recently proposed to deal with the blood vessel confounding effect in the IVIM-MRI model. This study is conducted using both realistic and real DW-MR images of human healthy liver of six volunteers (five males and one female) with age ranging from 23-28 years.

\subsection{Data acquisition and experiment setup}

The IVIM data are acquired from a 3 tesla GE MR scanner (GE Discovery MR 750, GEHC, Milwaukee, WI), employing an SE-EPI enhanced diffusion-weighted imaging (eDWI) sequence together with the array spatial sensitivity encoding technique (ASSET) for parallel imaging. The sequence parameters included $M=12 b$-values: 0,10 , $20,40,60,80,100,200,300,400,600$ and $800 \mathrm{sec} / \mathrm{mm}^{2}$ with repetition time of $2 \mathrm{sec}$, echo time of $54 \mathrm{~ms}$, Field Of View (FOV) of $40 \times 30 \mathrm{~cm}^{2}$, image matrix of $256 \times 256$ and slice thickness of $8 \mathrm{~mm}$. During the data acquisition, the volunteers were not required to hold breath. 
Numerical simulations are also conducted. the penalty parameters $\rho$ and $\lambda$, are chosen in a trial-and-error manner such that a smallest model error is obtained. Indeed, the model error is evaluated over a grid of $(\rho, \lambda)$-values with $(\rho, \lambda) \in\{0.1,0.2,0.5,1,10\} \times$ $\left\{10^{-8}, 10^{-6}, 10^{-4}, 10^{-2}, 1,10\right\}$, for both noise-free and noisy data and for different ROIs taken from the six volunteers in our data set. Regarding the noisy data configuration, three different values of the Signal-to-Noise Ratio (SNR) are considered (i.e., 50, 100 and 150). Note that, from an MR point of view, the SNR is defined as the ratio of the mean intensity of the chosen ROI to the standard deviation of the intensity outside the tissue (Lipton, 2008). According to our numerical experiments, the couple $(\rho, \lambda)$ that fulfils the smallest model error condition is $\left(0.2,10^{-6}\right)$ and hence it is retained for all subsequent experiments. It is worth noting that the quality of the identification results is highly dependent on the choice of the couple $(\rho, \lambda)$.
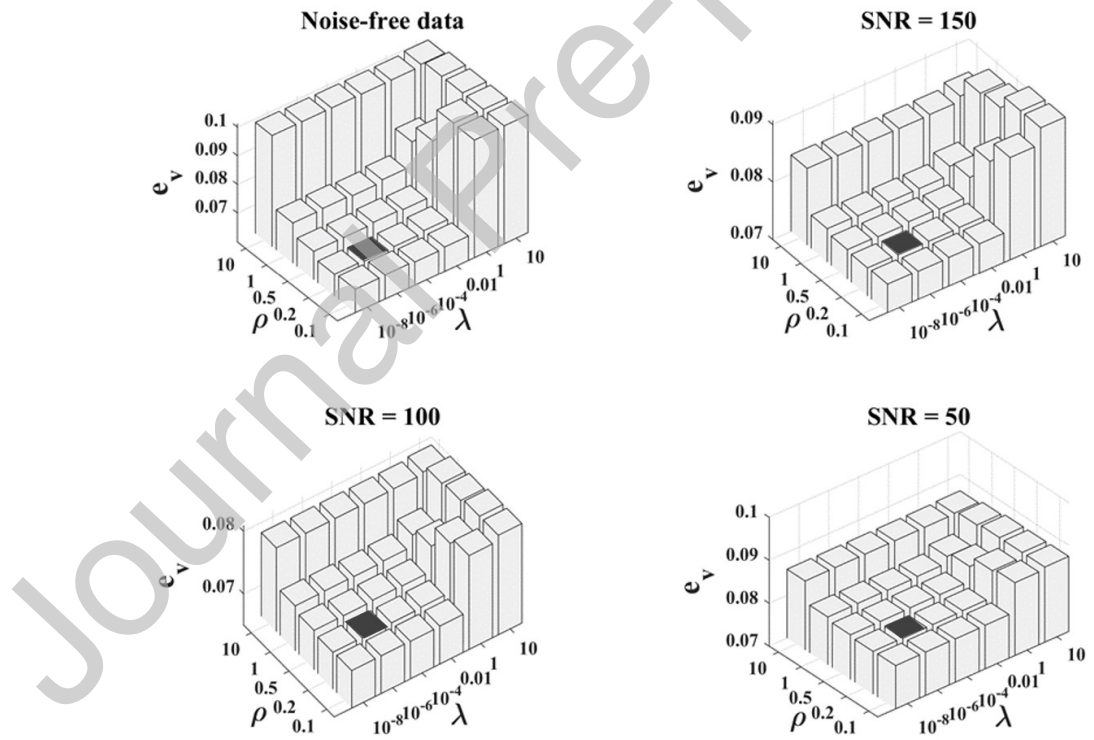

Fig. 1: The averaged model error per voxel over considered ROIs taken from the available six volunteers in our data set and over four noise levels (noise-free, SNR of 50,100 and 150), as a function of penalty parameters $\rho$ and $\lambda$. For noisy data, results are averaged over $25 \mathrm{MC}$ trials. The smallest model error is marked in dark square. 
To illustrate this fact, Figure 1 shows the model error per voxel, $e_{v}=\|\psi\|_{2} / N$ as a function of $(\rho, \lambda)$ values and for the aforementioned different situations of noise level (noise-free and noisy data with SNR values equal to 50,100 and 150). More precisely, the model error is evaluated for different realistic ROIs of size $(16 \times 16)$ taken from real MR images with a bifurcated blood vessel being simulated as shown in subsection 3.2. The model error is then averaged over the considered ROIs. Besides, for noisy data, results are also averaged over 25 Monte-Carlo (MC) trials. According to Figure 1, there is a set of $(\rho, \lambda)$ values for which the model error per voxel, $\boldsymbol{e}_{\nu}$, exhibits relatively small values. Among the latter set and whatever the noise level being studied here is, the smallest averaged model error per voxel is obtained for $(\rho, \lambda)=\left(0.2,10^{-6}\right)$, as illustrated in dark square.

\subsection{Realistic simulated data}

In order to fairly evaluate the behavior of the proposed SAVTE-IVIM $\mathrm{R}_{\mathrm{R}}$ and SAVTE$\mathrm{IVIM}_{\mathrm{E}}$ approaches, a ground truth is required. Therefore, for each volunteer, $M$ realistic noisy DW-MR images are generated from the $M$ available real ones by first performing conventional (voxel-by-voxel) bi-exponential fitting. Then, a third exponential decay effect with a very fast diffusion coefficient (i.e. $d_{3}^{(n)}, 1 \leq n \leq N$ ) ranging from 0.2 to $1 \mathrm{~mm}^{2} / \mathrm{sec}$, and amplitudes (i.e., $a_{3}^{(n)}, 1 \leq n \leq N$ ) ranging from $10 \%$ to $30 \%$ of the voxel intensities, is added to the chosen ROI. As shown in Fig. 2, the third component is added such that the considered ROI is affected by bifurcated blood vessel. Regarding the additive noise, random samples generated from a Rician probability distribution are used. Noise variance is adjusted to obtain the desired SNR value (Gudbjartsson and Patz, 2008). Numerical simulations are carried out, for each volunteer, over 25 MC trials and for different SNR values (50,100 and 150).

\subsubsection{Evaluation criteria}

As the main objective of the proposed SAVTE-IVIM approach is to identify blood vessels in the considered ROI, the identification quality is evaluated here in terms of the Voxel Localization Error (VLE) criterion, which provides a measure of similarity 

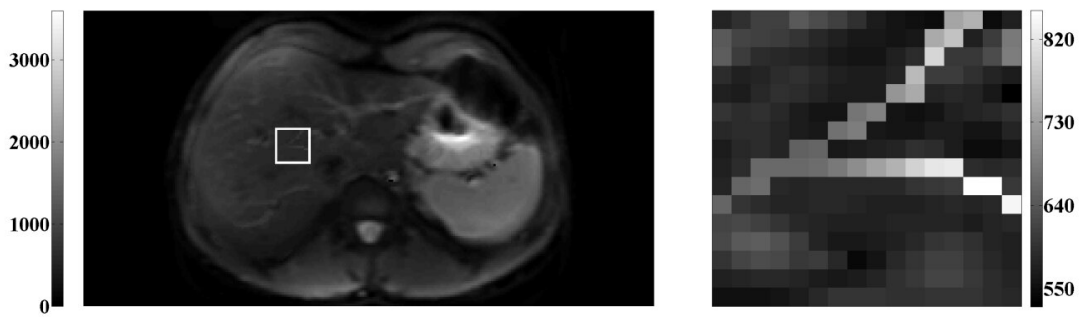

Fig. 2: Left: realistic DW-MR image (volunteer 1) at $b=0 \mathrm{sec} / \mathrm{mm}^{2}$ with parameters estimated from a bi-exponential fitting on a real DW-MR image of a liver in axial view. The ROI of size $(16 \times 16)$, marked in white square, contains a simulated bifurcating blood vessel. Right: a zoom-in of the considered ROI.

2017), the VLE is defined here as:

$$
V L E=\frac{1}{2 Q} \sum_{t \in \mathcal{I}} \min _{w \in \hat{I}}\left\|\mathbf{r}_{t}-\mathbf{r}_{w}\right\|_{2}+\frac{1}{2 \hat{Q}} \sum_{w \in \hat{I}} \min _{t \in \mathcal{I}}\left\|\mathbf{r}_{t}-\mathbf{r}_{w}\right\|_{2}
$$

where $\mathcal{I}$ and $\hat{\mathcal{I}}$ denote, respectively, the ground-truth and the estimated sets of indices of voxels affetcted by the third diffusion decaying component; $Q$ and $\hat{Q}$ are the cardinals of $\mathcal{I}$ and $\hat{\mathcal{I}}$, respectively; and $\mathbf{r}_{t}$ denotes the position of the $t$-th voxel. In addition to blood vessel identification, the SAVTE-IVIM allows not only for a quantification of the blood vessel contribution to the IVIM model but also for a quantification of the tissue diffusion and perfusion. Hence, the quantification quality of tissue diffusion and perfusion is evaluated here using the Normalized Mean Square Error (NMSE) defined by:

$$
\mathrm{NMSE}=\frac{\sum_{h=1}^{H}\left\|\mathbf{p}-\hat{\mathbf{p}}_{h}\right\|_{2}^{2}}{H\|\mathbf{p}\|_{2}^{2}}
$$

290 where $\mathbf{p}$ and $\hat{\mathbf{p}}_{h}$ denote, respectively, the ground-truth of the target parameter (ADC $\left(d_{1}\right), D^{*}\left(d_{2}\right)$ or PF) and its estimate computed at the $h$-th $(h \in\{1, \ldots, H\})$ MC trial.

\subsubsection{Blood vessel identification}

Since the NNLS-based algorithm (Gambarota et al., 2017) provides only an identi-

fication of the blood vessel confounding effect, the spatial distribution of $a_{3}$ is binary. Thus, for a fair comparison of the latter method with the proposed SAVTE-IVIM $_{R}$ and 
SAVTE-IVIM $\mathrm{E}_{\mathrm{E}}$ ones, which provide an identification and a quantification of the blood vessel confounding effect, a binary spatial distribution of $a_{3}$ obtained using the SAVTEIVIM $_{\mathrm{R}}$ and the SAVTE-IVIM $\mathrm{E}$ algorithms is created. To this end, the $\left(n_{1}, n_{2}\right)$-th voxel is labelled as affected when the estimated $a_{3}^{\left(n_{1}, n_{2}\right)}, 1 \leq n_{1} \leq N_{1}, 1 \leq n_{2} \leq N_{2}$ is higher than $10 \%$ of the voxel signal intensity. Figures 3 and 4 show the spatial distribution, $\boldsymbol{A}_{3}=$ unvec $\left(\boldsymbol{a}_{3}\right)$, of the blood vessel in the considered ROI for volunteers 1 and 2 in our data set.
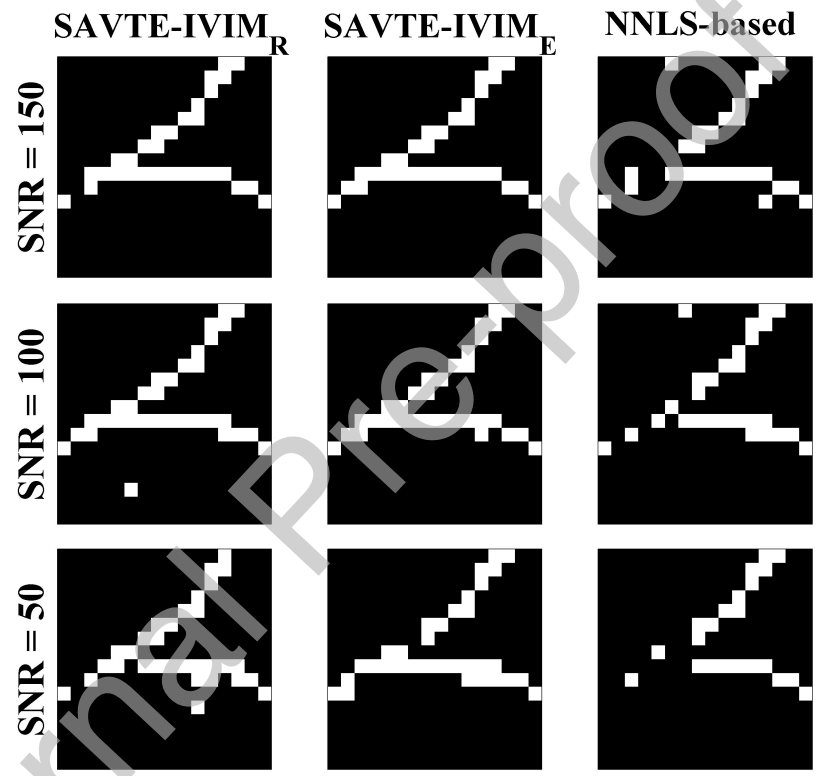

Fig. 3. The spatial distribution (binary map) of the very fast diffusion component, $a_{3}$ (corresponding to the blood vessel effect), for volunteer 1, as a function of the SNR and obtained using the NNLS-based (Gambarota

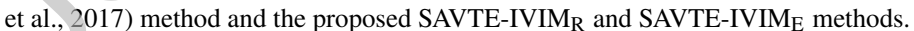



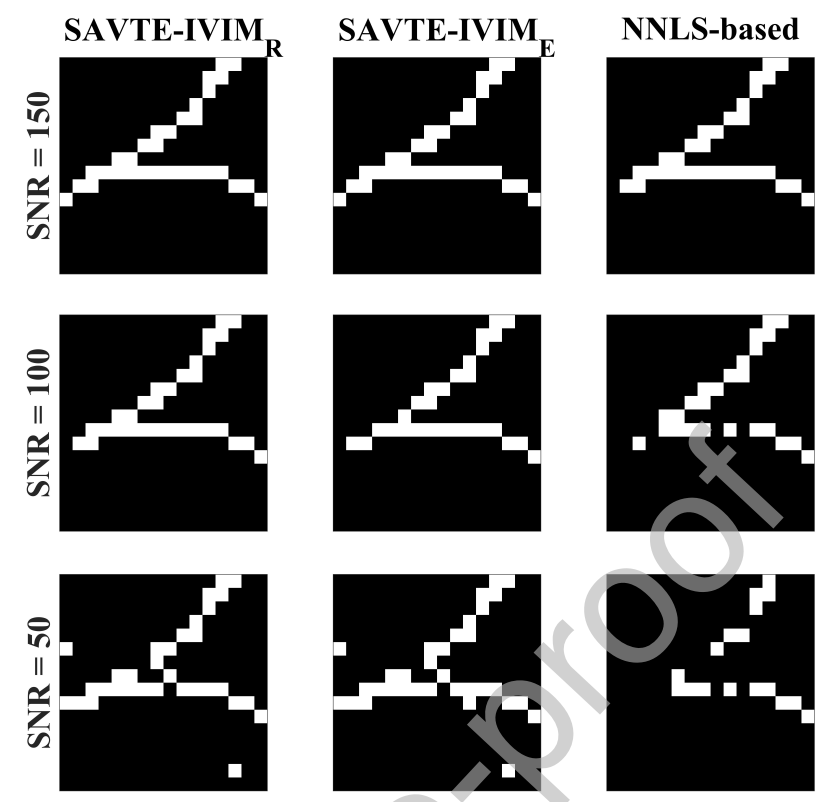

Fig. 4: The spatial distribution (binary map) of the very fast diffusion component, $a_{3}$ (corresponding to the blood vessel), for volunteer 2, as a function of the SNR and obtained using the NNLS-based (Gambarota et al., 2017) method and the proposed SAVTE-IVIM $\mathrm{R}$ and SAVTE-IVIME methods.

For the sake of readability, identification maps of blood vessels are presented here only for volunteer 1 and volunteer 2 while similar behavior of the considered algorithms was obtained for the other volunteers. This fact is confirmed in terms of VLE as shown in Figure 5. According to Figure 3 and Figure 4, the two proposed SAVTE-

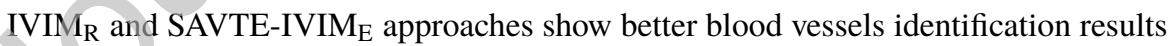
compared to the NNLS-based one for all SNR values. Indeed, for a relatively low SNR value ( $\mathrm{SNR}=50)$, the NNLS-based method (Gambarota et al., 2017) shows a high false negative rate in terms of identifying affected voxels while the proposed methods succeed in providing a localization map that is consistent with the ground truth. For higher SNR values (i.e., 100 and 150), better identification of blood vessels is to be noticed for the three considered methods, but with higher performance of the proposed approaches over the NNLS-based one (Gambarota et al., 2017). These results can also be confirmed using the VLE criterion (9) as depicted in Figure 5. Indeed, compared to 
the NNLS-based method (Gambarota et al., 2017), smaller VLE values are generally obtained using the proposed methods for all SNR values and for all volunteers in our data set.

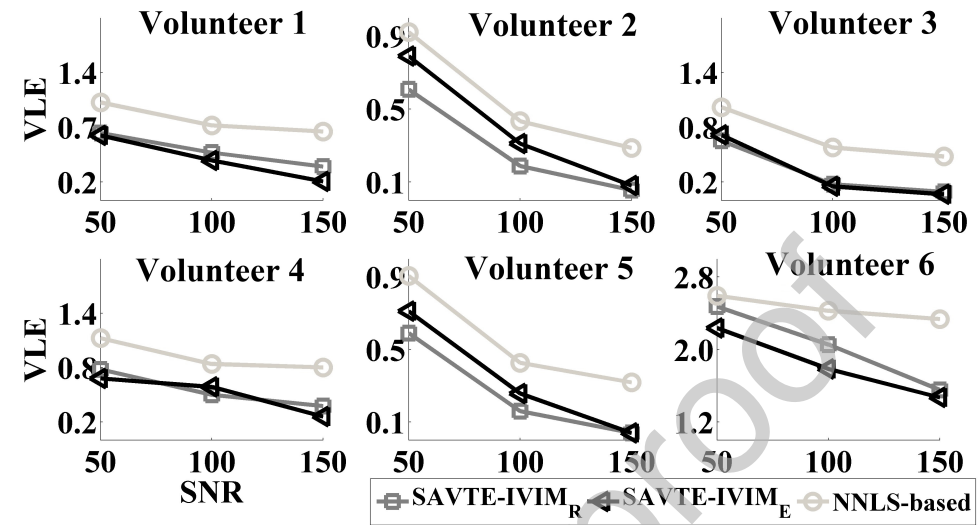

Fig. 5: VLE vs. SNR for the NNLS-based method (Gambarota et al,, 2017) and the proposed SAVTE-IVIM and SAVTE-IVIM $\mathrm{E}$ ones for six healthy volunteers. VLE values are averaged over $25 \mathrm{MC}$ trials.

\subsubsection{Quantification of tissue diffusion and perfusion}

In addition to the identification of blood vessels, the proposed SAVTE-IVIM approach allows to quantify the contribution of (i) detected blood vessels and (ii) the tissue perfusion and diffusion to the IVIM model. Figure 6 and Figure 7 show, for SNR $=50$, the parametric maps (i.e., the spatial distributions) of $\mathrm{ADC}\left(d_{1}\right), \mathrm{D}^{*}\left(d_{2}\right)$ and the $\mathrm{PF}$ for volunteer 1 and volunteer 2, respectively. Similar behavior is obtained for the other four volunteers in our data set. 

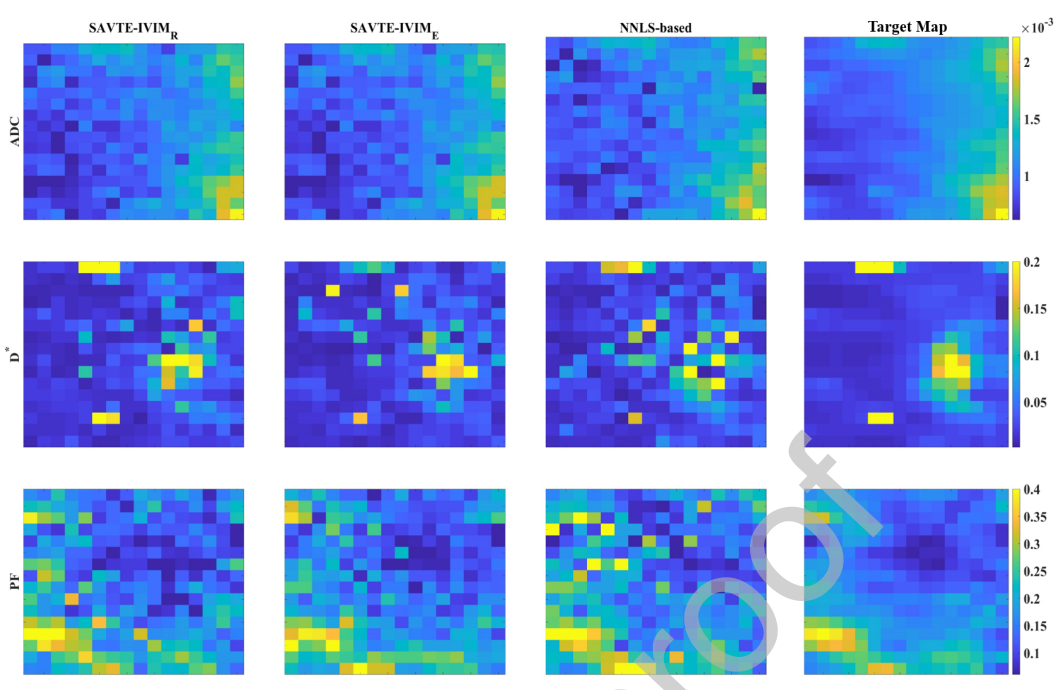

Fig. 6: Parametric maps of (i) the $\operatorname{ADC}\left(d_{1}, m^{2} / s e c\right)$ (top row), the pseudo-diffusion coefficient $\mathrm{D}^{*}\left(d_{2}\right.$, $\mathrm{mm}^{2} / \mathrm{sec}$ ) (middle row) and (iii) the perfusion fraction (bottom row), obtained using the SAVTE-IVIM , the SAVTE-IVIM $\mathrm{E}_{\mathrm{E}}$ and the NNLS-based methods for $\mathrm{SNR}=50$, (volunteer 1). 

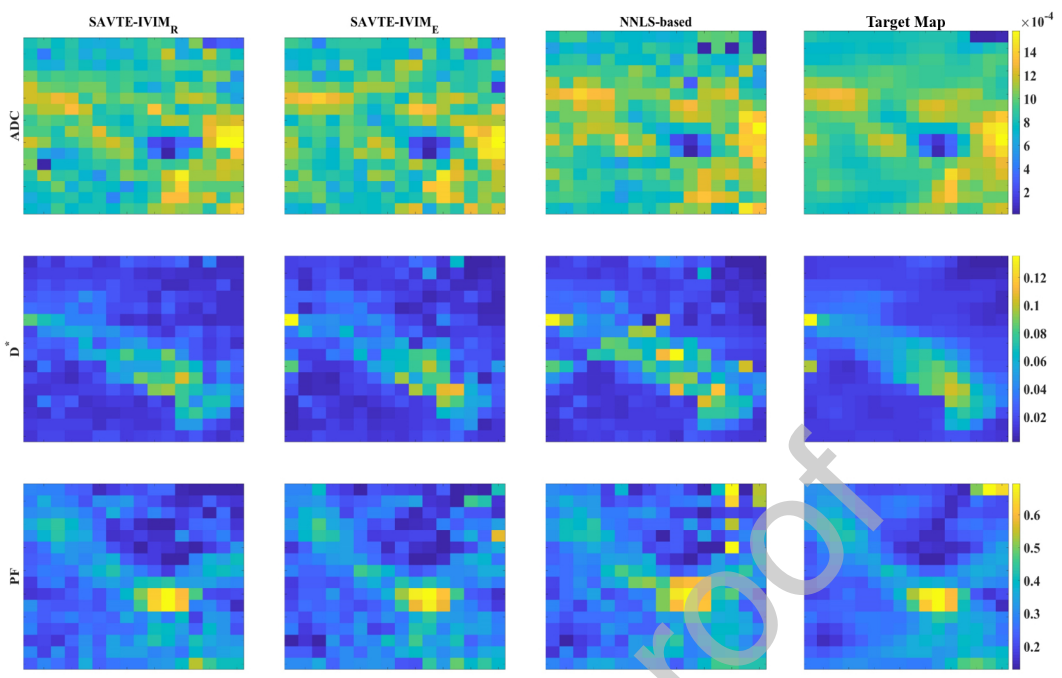

Fig. 7: Parametric maps of (i) the $\operatorname{ADC}\left(d_{1}, \mathrm{~mm}^{2} / \mathrm{sec}\right)$ (top row), (ii) the pseudo-diffusion coefficient $\mathrm{D}^{*}\left(d_{2}, \mathrm{~mm}^{2} / \mathrm{sec}\right)$ (middle row) and (iii) the perfusion fraction (bottom row), obtained using the SAVTEIVIM $_{R}$, the SAVTE-IVIME and the NNLS-based methods, for SNR=50 (volunteer 2).

According to Figure 6 and Figure 7, the three considered methods show generally comparable quantification results of $\operatorname{ADC}\left(d_{1}\right)$ and $\mathrm{D}^{*}\left(d_{2}\right)$ which are, to some extent, consistent with the target map. Regarding the quantification of PF, the NNLS-based method (Gambarota et al., 2017) shows, contrary to the proposed algorithms, a high number of spurious voxels with high PF values, as depicted in Figure 6 and Figure 7. This is mainly due to the fact that when the NNLS-based method (Gambarota et al., 2017) fails in detecting the presence of blood vessels, the PF is then calculated on the basis of the bi-exponential IVIM-MRI model. In this case, the blood vessel effect will contribute as a confounding factor to the estimation of $\mathrm{D}^{*}\left(d_{2}\right)$. As a result, higher values of $a_{2}$ are to be expected leading to high PF values in those voxels. It is worth noting that a similar behavior of the three considered methods was also observed for the quantification of $\operatorname{ADC}\left(d_{1}\right), \mathrm{D}^{*}\left(d_{2}\right)$ and $\mathrm{PF}$ in the other volunteers. As mentioned previously, the above quantification results can be evaluated also in terms of the NMSE

340 (10). Figure 8 shows the $\operatorname{ADC}\left(d_{1}\right), \mathrm{D}^{*}\left(d_{2}\right)$ and $\mathrm{PF}$ on a logarithmic scale as a function of SNR values for each volunteer and for the three considered methods, the 
SAVTE-IVIM $_{\mathrm{R}}$, the SAVTE-IVIM $\mathrm{E}_{\mathrm{E}}$ and the NNLS-based (Gambarota et al., 2017). Note that since the obtained NMSE values are generally smaller than one, computing the logarithm will lead to negative values. Therefore, for readability purposes, all NMSE values of $\operatorname{ADC}\left(d_{1}\right), \mathrm{D}^{*}\left(d_{2}\right)$ and $\mathrm{PF}$ were increased by one. We observe from Figure 8 that, for all considered parameters, better NMSE values are obtained when the SNR increases from 50 to 150 . Regarding the quantification of $\mathrm{ADC}\left(d_{1}\right)$, the proposed methods shows higher quantification quality compared to the NNLS-based method (Gambarota et al., 2017) for low $\mathrm{SNR}$ value $(\mathrm{SNR}=50)$ while comparable results are observed for the three considered methods for higher SNR values and for all volunteers in our data set except for volunteer 5 where the NNLS-based method shows better quantification of the $\operatorname{ADC}\left(d_{1}\right)$. Regarding the quantification of $\mathrm{D}^{*}\left(d_{2}\right)$, we note generally comparable results for the three methods, with a slight superiority of the SAVTE-IVIM $\mathrm{R}_{\mathrm{R}}$ over the SAVTE-IVIM $\mathrm{E}$ and the NNLS-based one (Gambarota et al., 2017). As far as the quantification of the PF is concerned, the SAVTE-IVIM $R$ and the SAVTE-IVIM $\mathrm{E}_{\mathrm{E}}$ generally outperform the NNLS-based one especially for low SNR. This behavior is confirmed for all volunteers in our data set except for volunteer 5 where the NNLS-based method shows relatively a higher quantification quality of the PF parameter. 

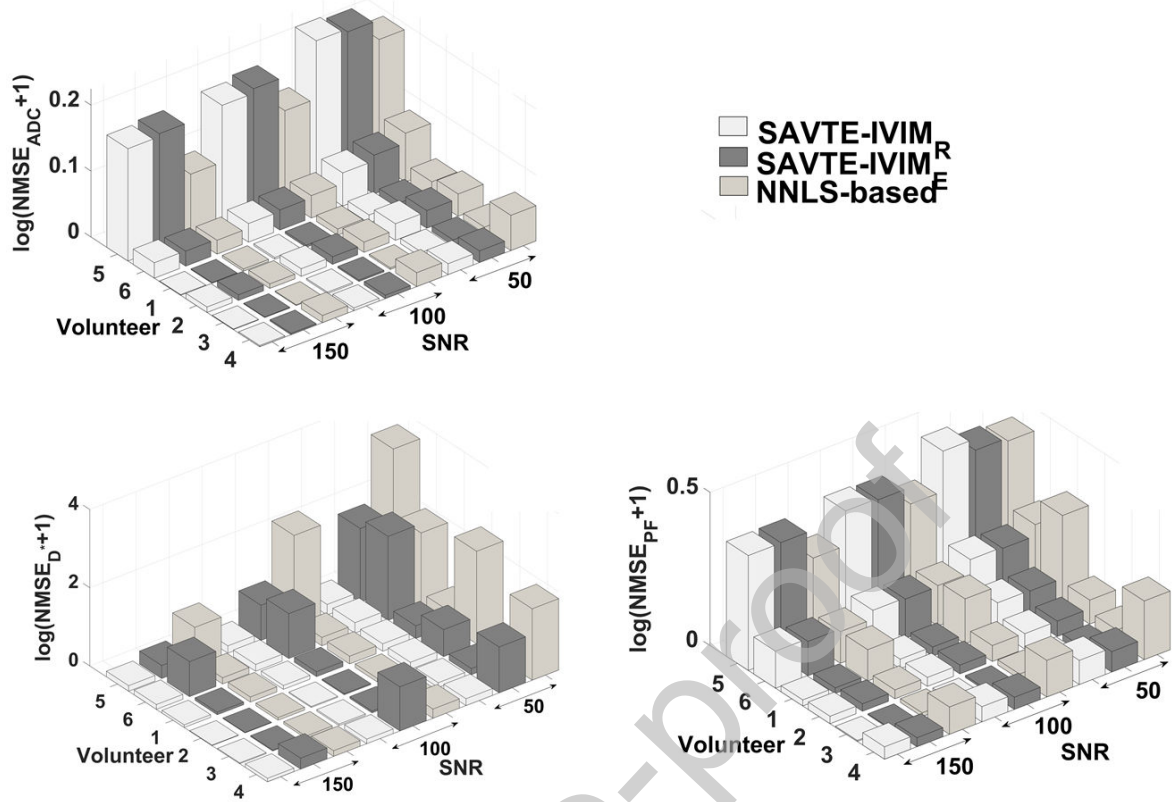

Fig. 8: Quantification of parameters ADC $\left(d_{1}\right), D^{*}\left(d_{2}\right)$ and PF in terms of NMSE using the NNLS-based method (Gambarota et al., 2017) and the proposed SAVTE-IVIM , the SAVTE-IVIM $_{E}$, for different SNR values $(150,100$ and 50) and for the six volunteers in our data set. For the sake of readability (i) the logarithm of NMSE values increased by one is considered and (ii) volunteers are irregularly ordered.

\subsubsection{Statistical Analysis}

In order to evaluate the statistical significance of the obtained results in the context of blood vessel identification and tissue diffusion/perfusion quantification, a Wilcoxon signed rank test is employed. More precisely, regarding the blood vessel identification part, a correlation series per method (e.g., the SAVTE-IVIM ${ }_{R}$, the SAVTE-IVIME and the NNLS-based (Gambarota et al., 2017)) is first constructed leading to three correlation series denoted here by $\gamma_{\mathrm{R}, \mathrm{TM}}, \boldsymbol{\gamma}_{\mathrm{E}, \mathrm{TM}}$ and $\gamma_{\mathrm{NNLS}, \mathrm{TM}}$. Each component of these series denotes the correlation coefficient computed, for one SNR value and for one volunteer, between a binarized version of the estimated spatial distribution of blood vessels in the considered ROI (see Figure 4) and a binarized version of the available Target Map (TM) depicted in Figure 2. Second, the Wilcoxon signed rank test is applied to each of the pairs $\left(\boldsymbol{\gamma}_{\mathrm{R}, \mathrm{TM}}, \boldsymbol{\gamma}_{\mathrm{E}, \mathrm{TM}}\right),\left(\boldsymbol{\gamma}_{\mathrm{R}, \mathrm{TM}}, \boldsymbol{\gamma}_{\mathrm{NNLS}, \mathrm{TM}}\right)$ and $\left(\boldsymbol{\gamma}_{\mathrm{E}, \mathrm{TM}}, \boldsymbol{\gamma}_{\mathrm{NNLS}, \mathrm{TM}}\right)$. 
Box plots of the differences $\boldsymbol{\gamma}_{\mathrm{R}, \mathrm{TM}}-\boldsymbol{\gamma}_{\mathrm{E}, \mathrm{TM}}, \boldsymbol{\gamma}_{\mathrm{R}, \mathrm{TM}}-\boldsymbol{\gamma}_{\mathrm{NNLS}, \mathrm{TM}}$ and $\boldsymbol{\gamma}_{\mathrm{E}, \mathrm{TM}}-\boldsymbol{\gamma}_{\mathrm{NNLS}, \mathrm{TM}}$ are shown in Figure 9 together with the corresponding p-values (shown in a box). Compared to the NNLS-based approach (Gambarota et al., 2017), blood vessel identification results obtained using both the proposed SAVTE-IVIM $\mathrm{R}_{\mathrm{R}}$ and the SAVTE-IVIM $\mathrm{E}$ algorithms are of high statistical significance (p-values of $2.3 \times 10^{-3}$ and $1.9 \times 10^{-3}$, respectively). Furthermore, as expected, no statistical difference between the results of the SAVTEIVIM $_{E}$ and those of the SAVTE-IVIM $R$ is observed (p-value of 0.91). This is since the two methods show generally similar blood vessel identification results.

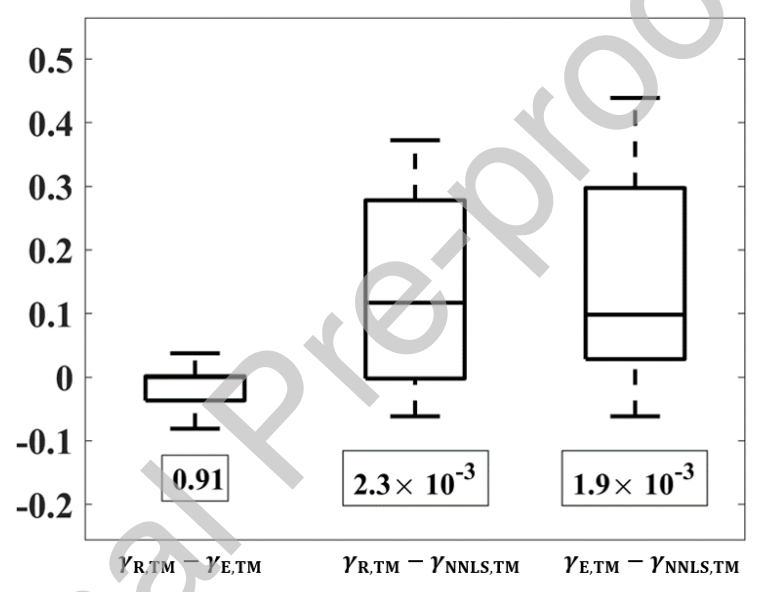

Fig. 9: Box plots of the difference between paired correlation series $\boldsymbol{\gamma}_{\mathrm{R}, \mathrm{TM}}, \boldsymbol{\gamma}_{\mathrm{E}, \mathrm{TM}}$ and $\boldsymbol{\gamma}_{\mathrm{NNLS}, \mathrm{TM}}$ used for the Wilcoxon signed rank test. Obtained p-values are presented in boxes.

Regarding the quantification of tissue diffusion and perfusion, specifically the parameter $\mathrm{ADC}\left(d_{1}\right), \mathrm{PF}$ and $\mathrm{D}^{*}\left(d_{2}\right)$, a correlation series per parameter is built leading to $\gamma_{\mathrm{R}, \mathrm{TM}}^{\theta}, \gamma_{\mathrm{E}, \mathrm{TM}}^{\theta}, \gamma_{\mathrm{NNLS}, \mathrm{TM}}^{\theta}$ with $\theta \in\left\{\mathrm{ADC}, \mathrm{PF}, \mathrm{D}^{*}\right\}$. For example, each element of $\gamma_{\mathrm{R}, \mathrm{TM}}^{\mathrm{ADC}}$ denotes the correlation coefficient computed, for one ROI, between the true parametric map (the target map) related to the ADC and its estimate using the SAVTE-IVIM $_{R}$ method (see Figures 6 and 7). Then, a Wilcoxon signed rank test is applied to the three pairs of correlation series $\left(\gamma_{\mathrm{R}, \mathrm{TM}}^{\theta}, \gamma_{\mathrm{E}, \mathrm{TM}}^{\theta}\right),\left(\gamma_{\mathrm{R}, \mathrm{TM}}^{\theta}, \gamma_{\mathrm{NNLS}, \mathrm{TM}}^{\theta}\right)$ and $\left(\gamma_{\mathrm{E}, \mathrm{TM}}^{\theta}, \gamma_{\mathrm{NNLS}, \mathrm{TM}}^{\theta}\right), \theta \in\left\{\mathrm{ADC}, \mathrm{PF}, \mathrm{D}^{*}\right\}$. Box plots of the differences $\gamma_{1}^{\theta}=$ 
$\gamma_{\mathrm{R}, \mathrm{TM}}^{\theta}-\gamma_{\mathrm{E}, \mathrm{TM}}^{\theta}, \gamma_{2}^{\theta}=\gamma_{\mathrm{R}, \mathrm{TM}}^{\theta}-\gamma_{\mathrm{NNLS}, \mathrm{TM}}^{\theta}$ and $\gamma_{3}^{\theta}=\gamma_{\mathrm{E}, \mathrm{TM}}^{\theta}-\gamma_{\mathrm{NNLS}, \mathrm{TM}}^{\theta}$ are shown in Figure 10 together with the corresponding p-values (shown in boxes). According to latter figure, we note that (i) regarding the $\operatorname{ADC}\left(d_{1}\right)$ quantification: all methods show comparable results. This fact is also confirmed by the obtained p-values (0.94 and 0.96); (ii) regarding the PF quantification: all methods show again comparable results as also confirmed by the obtained p-values (0.99 and 0.96) and (iii) regarding the $D^{*}\left(d_{2}\right)$ quantification: compared to the NNLS-based approach (Gambarota et al., 2017), the SAVTE-IVIM $\mathrm{E}_{\mathrm{E}}$ algorithm shows higher statistical significance (i.e. p-value of $1.5 \times 10^{-4}$ ) while no statistical significance is to be noted from the SAVTE-IVIM $\mathrm{R}_{\mathrm{R}}$ (i.e. p-value of 0.55).
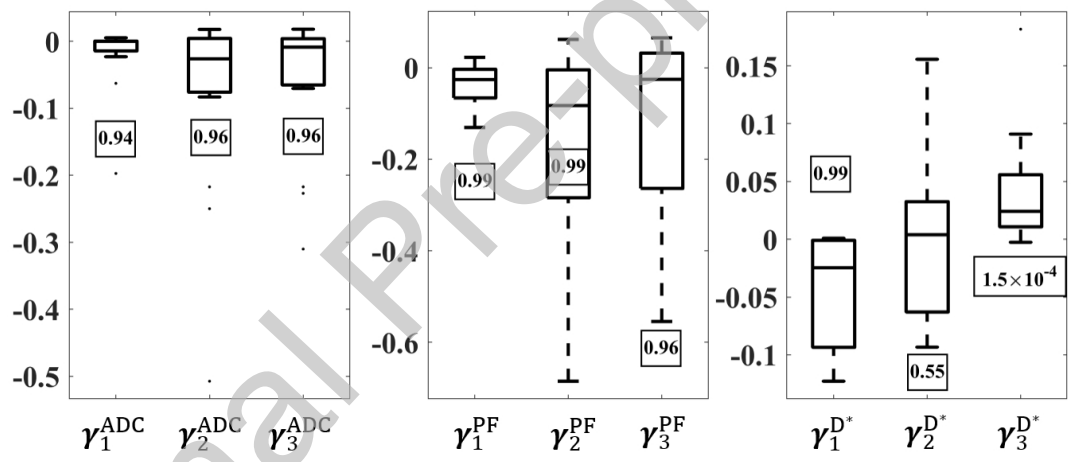

Fig. 10: Box plots of the difference between paired correlation series $\gamma_{1}^{\theta}=\gamma_{\mathrm{R}, \mathrm{TM}}^{\theta}-\gamma_{\mathrm{E}, \mathrm{TM}}^{\theta}, \gamma_{2}^{\theta}=$ $\gamma_{\mathrm{R}, \mathrm{TM}}^{\theta}-\gamma_{\mathrm{NNLS}, \mathrm{TM}}^{\theta}$ and $\boldsymbol{\gamma}_{3}^{\theta}=\gamma_{\mathrm{E}, \mathrm{TM}}^{\theta}-\gamma_{\mathrm{NNLS}, \mathrm{TM}}^{\theta}$ for $\theta \in\left\{\mathrm{ADC}, \mathrm{PF}, \mathrm{D}^{*}\right\}$ used for the Wilcoxon signed rank test. Obtained $\mathrm{p}$-values are presented in boxes.

\subsection{Real data}

To explore the feasibility of the considered algorithms on a real data set, two comparative studies are considered hereafter. First, the behavior of these algorithms is investigated, for a given volunteer (i.e., volunteer 1) in our data set, as a function of the chosen ROI. Second, these methods are evaluated using the DW-MR images of the other volunteers (e.g., volunteer 2, 3, 4, 5 and 6 ) where only one informative ROI per volunteer is considered. The ROI selection is performed in the following way: 
first, regions where macroscopic blood vessels are present, as assessed by direct visual inspection of the images, are excluded. Indeed, these areas where the blood vessel confounding effect is evident are typically excluded by clinicians when evaluating tissue diffusion and perfusion. Secondly, the image contrast of the DW-MR image at $b=0 \mathrm{sec} / \mathrm{mm}^{2}$ is manually enhanced, to reach nearly saturation levels. This step is performed in order to identify areas characterized by a limited number of voxels that display a higher signal w.r.t. to neighboring voxels; this is indicative of partial volume between blood vessels and liver parenchyma (Gambarota et al., 2017).

Regarding the first study, four ROIs of size $(16 \times 16)$ are chosen from volunteer 1 using manually enhanced image contrast. Figure 11(a) shows the real DW-MR image (top left) with a standard contrast of a human liver. A zoom-in of these four chosen ROIs (white squares) is shown in Figure 11(b)-(e).

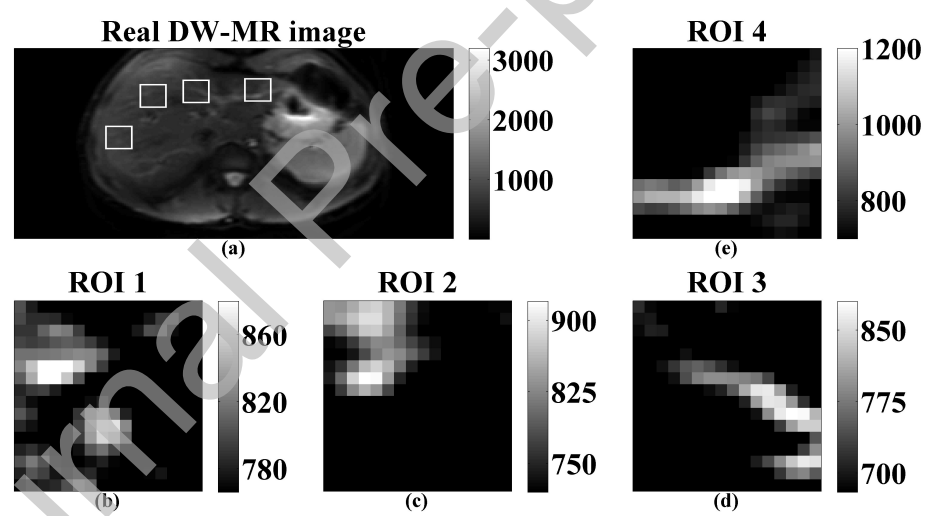

Fig. 11: (a) Real DW-MR image of a liver (volunteer 1) in axial view. Four ROIs (white squares) of size $(16 \times 16)$ are chosen (from left to right, ROI 1 to ROI 4). (b)-(e) Zoom-in images (ROI 1 to ROI 4) shown with manually enhanced image contrast.

It should be noted that the behavior of the manual enhancement of image contrast is operator-dependent. To illustrate this fact, the ROI 4 is shown in Figure 12 with three different contrast levels leading to three different visualization aspects. Thus, the contrast-enhancing approach can not be employed as an unbiased mean to identify blood vessels. 

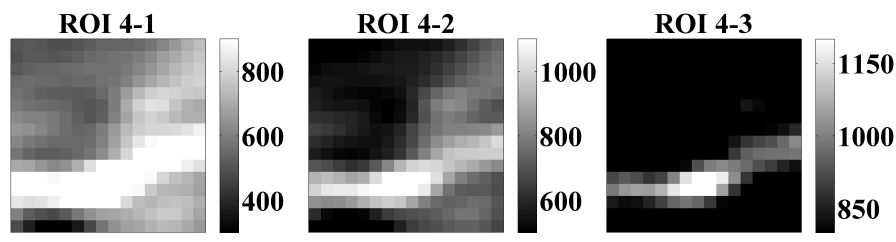

Fig. 12: Three zoom-in images of ROI 4 shown in different contrast levels.

Figure 13 shows the spatial distribution (i.e., $\boldsymbol{A}_{3}=\operatorname{unvec}\left(\boldsymbol{a}_{3}\right)$ ) of the identified third diffusion component (the blood vessel effect) using the SAVTE-IVIM $\mathrm{R}_{\mathrm{R}}$, the SAVTEIVIM $_{\mathrm{E}}$ and the NNLS-based (Gambarota et al., 2017) methods.

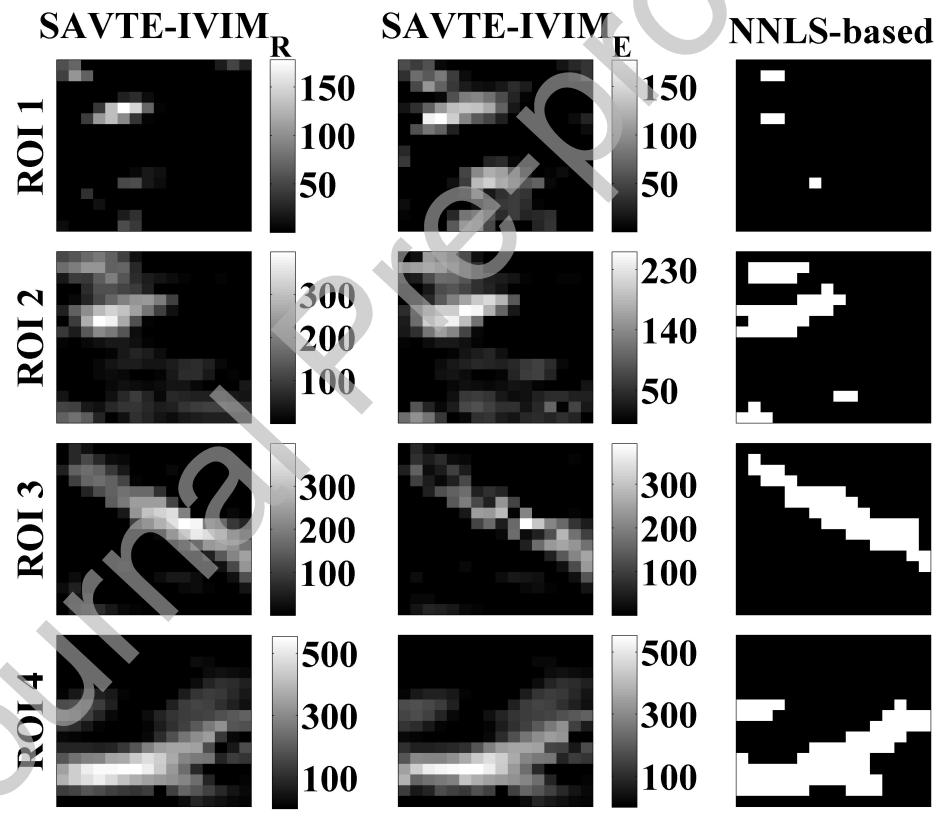

Fig. 13: The spatial distribution of blood vessels quantified using the SAVTE-IVIM $_{R}$, the SAVTE-IVIM and the NNLS-based (Gambarota et al., 2017) methods for four different ROIs.

${ }_{425}$ We stress on the fact that contrary to the NNLS-based approach wherein the provided spatial distribution of this third exponential component is a binary map, the proposed approaches result in simultaneous identification and quantification of this component. Variations of the contrast level in the spatial distribution of $a_{3}$ obtained using the 
SAVTE-IVIM $_{\mathrm{R}}$ and the SAVTE-IVIM $\mathrm{E}_{\mathrm{E}}$ algorithms confirm this fact. Regarding the 430 quantification of $\mathrm{ADC}\left(d_{1}\right), \mathrm{D}^{*}\left(d_{2}\right)$ and $\mathrm{PF}$ shown in Table 1 , the three methods show competitive results which are consistent with the values found in previous studies (Gambarota et al., 2017; Barbieri et al., 2016; Leporq et al., 2015).

Table 1: Quantification of the $\operatorname{ADC}\left(d_{1}\right)$, the PF and the pseudo-diffusion coefficient $\mathrm{D}^{*}\left(d_{2}\right)$ using the SAVTEIVIM $_{R}$, the SAVTE-IVIM $\mathrm{E}$ and the NNLS-based methods for four different ROIs chosen from volunteer 1. The parameters ADC, PF and $\mathrm{D}^{*}$ are expressed, respectively, in $\left(\times 10^{-4} \mathrm{~mm}^{2} / \mathrm{sec}\right),(\%)$ and $\left(\mathrm{mm}^{2} / \mathrm{sec}\right)$

\begin{tabular}{|c|c|c|c|}
\hline ADC & SAVTE-IVIM $_{\mathrm{R}}$ & SAVTE-IVIM $_{\mathrm{E}}$ & NNLS-based \\
\hline ROI 1 & $9.0 \pm 1.3$ & $8.8 \pm 1.2$ & $8.6 \pm 1.8$ \\
\hline ROI 2 & $9.7 \pm 1.2$ & $9.6 \pm 1.2$ & $9.2 \pm 1.6$ \\
\hline ROI 3 & $11.0 \pm 2.1$ & $11.0 \pm 2.1$ & $10.0 \pm 2.5$ \\
\hline ROI 4 & $8.6 \pm 7.5$ & $9.0 \pm 8.9$ & $8.6 \pm 8.2$ \\
\hline \hline PF & SAVTE-IVIM & SAVTE-IVIM $_{\mathrm{E}}$ & NNLS-based \\
\hline ROI 1 & $19.2 \pm 9.1$ & $24.4 \pm 7.4$ & $27.4 \pm 8.8$ \\
\hline ROI 2 & $13.7 \pm 7.7$ & $15.2 \pm 6.2$ & $19.5 \pm 9.5$ \\
\hline ROI 3 & $24.9 \pm 13.3$ & $26.7 \pm 11.5$ & $31.3 \pm 13.2$ \\
\hline ROI 4 & $49.1 \pm 11.8$ & $49.5 \pm 10.5$ & $59.0 \pm 10.0$ \\
\hline \hline D* & SAVTE-IVIM & SAVTE-IVIM & NNLS-based \\
\hline ROI 1 & $0.057 \pm 0.022$ & $0.022 \pm 0.018$ & $0.024 \pm 0.018$ \\
\hline ROI 2 & $0.047 \pm 0.053$ & $0.031 \pm 0.034$ & $0.029 \pm 0.031$ \\
\hline ROI 3 & $0.048 \pm 0.046$ & $0.038 \pm 0.037$ & $0.034 \pm 0.035$ \\
\hline ROI 4 & $0.023 \pm 0.036$ & $0.019 \pm 0.026$ & $0.021 \pm 0.025$ \\
\hline
\end{tabular}

Note that since no constraints regarding the physiological values of $\mathrm{D}^{*}$ are to be considered neither in the SAVTE-IVIM $\mathrm{E}_{\mathrm{E}}$ algorithm nor in the NNLS-based one, some estimates of $\mathrm{D}^{*}$, for certain voxels, are expected to be higher than its largest physiological value (e.g., 0.2). In such situation, corresponding voxels are excluded from the subsequent within-ROI averaging operations, as suggested in (Barbieri et al., 2016).

As far as the second study is concerned, for each of the five volunteers (i.e., volunteer 2, 3, 4, 5 and 6), one informative ROI is selected (Figure 14, first column). The 

contrast enhancement (Figure 14, second column).

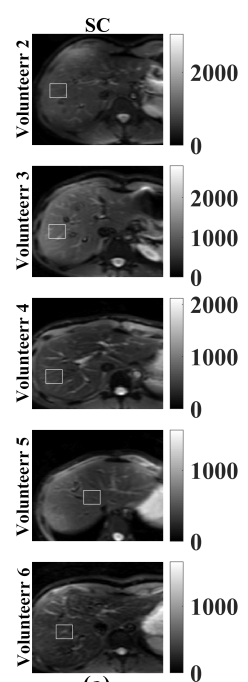

(a)
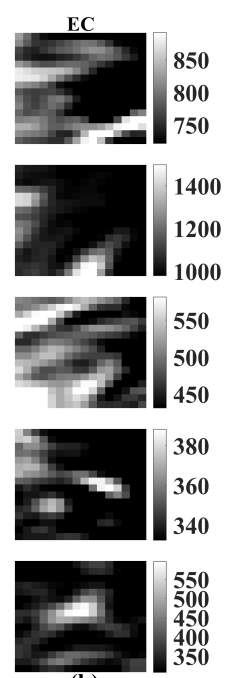

(b)
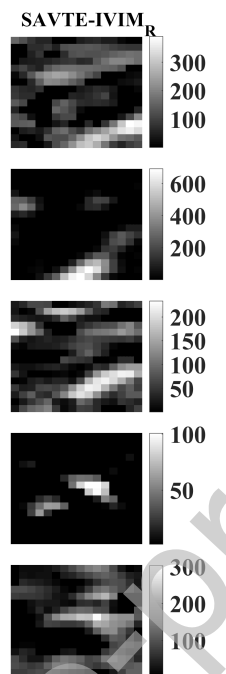

(c)
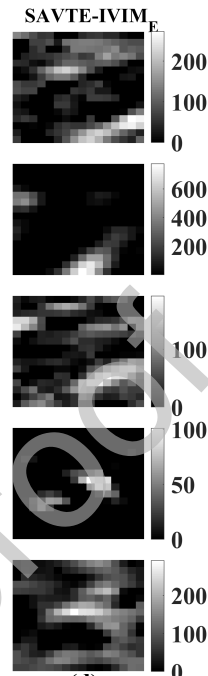

(d)
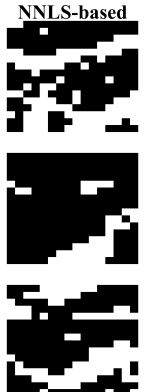

צ

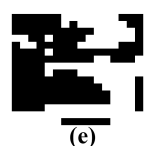

Fig. 14: (a) Selected ROIs (white squares) from five volunteers (one ROI per volunteer) shown with Standard Contrast (SC), (b) a zoom-in of ROIs shown in manually Enhanced Contrast (EC), (c-e) estimated spatial distribution maps of blood vessels using the SAVTE-IVIM , the SAVTE-IVIM $_{\mathrm{E}}$ and the NNLS-based methods (Gambarota et al., 2017), respectively.

The spatial distribution of blood vessels (e.g., $a_{3}$ ) in each ROI using the SAVTEIVIM $_{R}$, the SAVTE-IVIM ${ }_{E}$ and the NNLS-based methods is depicted in Figure 14. We note that while the NNLS-based approach provides only a binary map reflecting only an identification of blood vessels, the proposed algorithms provide simultaneous identification and quantification of blood vessels. Furthermore, we note from Figure 14 that all methods generally succeed in identifying voxels which seem to be affected by blood vessels. However, the NNLS-based approach provides generally a spatial distribution of blood vessels that is, to some extent, less consistent with the available indicative distribution of blood vessels. For instance, compared to the latter, in the case of volunteer 5, the NNLS-based method seems to provide a higher false negative identification rate than the proposed algorithms. Regarding the quantification of ADC $\left(d_{1}\right), \mathrm{D}^{*}\left(d_{2}\right)$ and PF, Table 2 shows that the three considered methods provide generally, 
for the five volunteers, quantification results that are consistent with those found in previous studies on human liver (Gambarota et al., 2017; Barbieri et al., 2016; Leporq et al., 2015).

Table 2: Quantification of the ADC, the PF and the $\mathrm{D}^{*}$ using the SAVTE-IVIM $\mathrm{R}_{\mathrm{R}}, \mathrm{SAVTE}_{\mathrm{IVIM}}$ and the NNLS-based methods for volunteers 2-6. ADC, PF and $\mathrm{D}^{*}$ are expressed, respectively, in $\left(\times 10^{-4} \mathrm{~mm}^{2} / \mathrm{sec}\right)$, (\%) and $\left(\mathrm{mm}^{2} / \mathrm{sec}\right)$

\begin{tabular}{|c|c|c|c|}
\hline ADC & SAVTE-IVIM $_{\mathrm{R}}$ & SAVTE-IVIM $_{\mathrm{E}}$ & NNLS-based \\
\hline Volunteer 2 & $10.1 \pm 1.6$ & $10.4 \pm 1.4$ & $4.6 \pm 4.7$ \\
\hline Volunteer 3 & $10.8 \pm 1.4$ & $10.8 \pm 1.4$ & $10.8 \pm 1.6$ \\
\hline Volunteer 4 & $9.6 \pm 3.4$ & $9.8 \pm 3.0$ & $7.9 \pm 2.8$ \\
\hline Volunteer 5 & $16.2 \pm 2.2$ & $16.5 \pm 2.1$ & $15.1 \pm 4.6$ \\
\hline Volunteer 6 & $12.7 \pm 2.1$ & $12.8 \pm 2.0$ & $12.8 \pm 2.3$ \\
\hline \hline PF & SAVTE-IVIM & SAVTE-IVIM & NNLS-based \\
\hline Volunteer 2 & $9.8 \pm 7.5$ & $12.7 \pm 4.2$ & $44.3 \pm 24.3$ \\
\hline Volunteer 3 & $16.1 \pm 6.0$ & $16.5 \pm 4.1$ & $19.9 \pm 15.5$ \\
\hline Volunteer 4 & $11.7 \pm 9.7$ & $14.1 \pm 7.3$ & $24.3 \pm 16.9$ \\
\hline Volunteer 5 & $20.3 \pm 10.4$ & $19.5 \pm 9.7$ & $26.0 \pm 17.7$ \\
\hline Volunteer 6 & $30.8 \pm 12.8$ & $29.1 \pm 10.7$ & $41.5 \pm 14.1$ \\
\hline \hline D* & SAVTE-IVIM & SAVTE-IVIM & NNLS-based \\
\hline Volunteer 2 & $0.109 \pm 0.073$ & $0.075 \pm 0.065$ & $0.093 \pm 0.040$ \\
\hline Volunteer 3 & $0.130 \pm 0.031$ & $0.126 \pm 0.029$ & $0.125 \pm 0.031$ \\
\hline Volunteer 4 & $0.089 \pm 0.067$ & $0.076 \pm 0.060$ & $0.055 \pm 0.055$ \\
\hline Volunteer 5 & $0.036 \pm 0.022$ & $0.037 \pm 0.021$ & $0.038 \pm 0.025$ \\
\hline Volunteer 6 & $0.073 \pm 0.056$ & $0.063 \pm 0.044$ & $0.068 \pm 0.047$ \\
\hline
\end{tabular}

However, the latter table shows some relatively higher PF values using the NNLS-based method compared to the ones obtained using the SAVTE-IVIM $\mathrm{E}_{\mathrm{E}}$ and the SAVTE$\mathrm{IVIM}_{\mathrm{R}}$ algorithms. This is probably due, as discussed previously, to the blood vessel confounding effect that systematically appears when a bi-exponential IVIM model fitting is to be considered for each voxel where a false negative identification of blood 
vessel has occurred.

\section{Discussion}

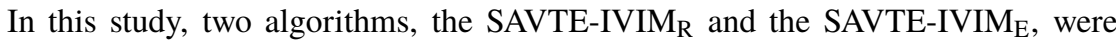
proposed to deal with the problem of the confounding blood vessel effect in the IVIMMRI model in liver. These algorithms were evaluated and compared to the NNLSbased one recently proposed to deal with such problem. Our comparative study was conducted using simulated and real DW-MR images of liver acquired from six healthy volunteers. The results on simulated data showed the superiority of the proposed methods over the NNLS-based one in terms of blood vessel identification. Indeed, the NNLS-based method showed higher false negative rate in terms of identifying affected voxels, especially for low SNR, as confirmed by the VLE measure and by the statistical analysis. In terms of quantifying $\operatorname{ADC}\left(d_{1}\right)$ and $\mathrm{D}^{*}\left(d_{2}\right)$, all methods showed competitive results. However, the proposed algorithms showed higher performance in terms of quantifying PF. In fact, contrary to the NNLS-based method, the SAVTEIVIM $_{R}$ and the SAVTE-IVIM $M_{\mathrm{E}}$ methods provided PF maps that are more consistent with the ground truth. Furthermore, a high rate of spurious voxels with high PF values were observed especially for NNLS-based method. This is mainly due to the false negative identification of affected voxels. More precisely, for a false negative detection of affected voxels, a bi-exponential fitting instead of a tri-exponential one is to be systematically considered in the NNLS-based method. Indeed, with the bi-exponential fitting being employed, the confounding blood vessel effect will highly contribute to the estimation of the tissue perfusion. Consequently, high PF values are to be expected. Regarding the experiments on real DW-MR images, all methods gave, to some extent, comparable spatial distribution of blood vessels in the ROI. However the NNLS-based one showed higher PF quantification values compared to the ones obtained using the proposed methods. This phenomena is, as discussed above, mainly due to the problem of false negative identification of affected voxels. Finally, it is noteworthy that as the spatial distribution of blood vessel in liver is not uniform, there exists some ROIs for which the sparsity assumption of blood vessels on which the proposed approach relies, 
is fulfilled. Besides, it is worth noting that the sparsity rate is highly dependent on the size of the chosen ROI. For example, the sparsity assumption can easily be violated for small ROIs ( i.e., $2 \times 2$ ). But, as the typical size of an ROI delineated by clinical expert is, in general, not less than $(10 \times 10)$, one can always find an ROI for which the sparsity assumption on the blood vessel spatial distribution holds true. In such case the defined ROI is well-called informative.

Besides, evaluating the proposed algorithm on real DW-MR images is still a hard task since the ground truth regarding the distribution of blood vessel in the ROI under study is generally unavailable. To cope with this issue, additional MR images such as the MR angiogram could be employed to provide a ground truth in vivo. However it should be noted that: (i) in the current work we investigate the case of a small $(10-20 \%)$ partial volume effect between blood vessels and liver parenchyma; (ii) the IVIM DW-MRI protocol consists typically of data acquisition in free-breathing over a few minutes; (iii) DW-MR images need to be co-registered with those of the MR angiogram. Due to this intrinsic limitation of the co-registration process between free-breathing images acquired on a moving organ in two separate measurements, the approach of an MR angiogram as a reference ground truth for small $(10-20 \%)$ partial volume effects was discarded in current work. Further studies would be necessary to investigate in detail this challenging strategy

\section{Conclusion}

In this paper, a new approach to cope with the confounding blood vessel effect in the IVIM-MRI model in liver was proposed. This is accomplished by resorting to an automatic identification of the potential presence of large blood vessels in the ROI, especially in situations where visual identification of such blood vessels is not evident.

Based on (i) the sparsity assumption of the spatial distribution of large vessels in the ROI and (ii) the extension of the single voxel-wise IVIM-MRI model to the all-voxel wise one, the main advantage of the proposed SAVTE-IVIM approach resides in its ability to deal simultaneously with all voxels in the ROI. In addition, two different strategies have been proposed to take into account the non-negativity constraints in the all-voxel tri- 
exponential IVIM-MRI model: (i) a rough strategy, where potential negative values of parameter estimates were dealt with by taking into account prior informations regarding the physiological ranges of the model parameters; and (ii) an embedded strategy where a change of variable was employed during the optimization process. In addition to the automatic blood-vessel identification, the SAVTE-IVIM approach, in its two proposed variants, provides a quantification of (i) the confounding blood vessel effect of each voxel; (ii) the apparent diffusion coefficient and (iii) the tissue perfusion. Our results on both realistic and real DW-MR images of six healthy volunteers have shown the efficiency of the proposed algorithm. Indeed, the two proposed algorithms SAVTEIVIM $_{R}$ and SAVTE-IVIM ${ }_{E}$ have shown generally comparable performances in terms of blood vessel identification and quantification of tissue diffusion and perfusion. In the case where the prior information regarding the physiological range of the model parameters is reliable, it is preferable to use the SAVTE-IVIM $\mathrm{R}_{\mathrm{R}}$ method.

\section{Acknowledgment}

The authors gratefully acknowledge the financial support from the China Scholarship Council. Part to this study was conducted within the LABEX PRIMES (ANR11-LABX-0063) of the "Universite de Lyon" from the "Investissements d'Avenir" program (ANR-11-IDEX-0007) operated by the French National Research Agency (ANR). This work was also supported in part by the National Key R\&D Program of China (2017YFC107900) and in part by the Excellence Project Funds of Southeast University.

\section{so Appendices}

\section{A. Technical materials on the solution of the P3 problem (6)}

As mentioned in section 2.2.1, the ADMM method (Boyd et al., 2011) is used to solve the $\mathrm{P} 2$ problem (5). To do so, the latter is reformulated in terms of minimizing its associated augmented Lagrangian function , $\mathcal{L}$, leading to the P3 problem (6) recalled 
hereafter:

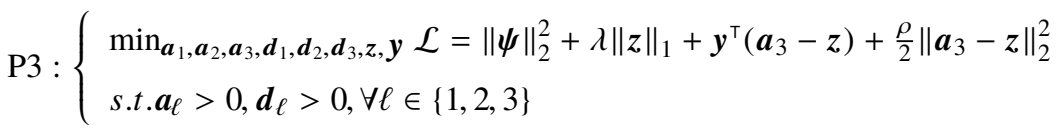

where $z$ is an $N$-dimensional latent variable, $\boldsymbol{y} \in \mathbb{R}^{N}$ is the Lagrangian multiplier and $\rho>0$ denotes a penalty parameter. Estimating $\boldsymbol{a}_{\ell}, \forall \ell\{1,2,3\}$ can be easily computed in a least square sense as the solution of $\frac{\partial \mathcal{L}}{\partial \boldsymbol{a}_{\ell}}=0, \forall \ell \in\{1,2,3\}$. This leads to:

$$
\begin{aligned}
& \boldsymbol{a}_{1}=-\left(\boldsymbol{J}_{\boldsymbol{a}_{1}}^{\top} \boldsymbol{J}_{\boldsymbol{a}_{1}}\right)^{-1} \boldsymbol{J}_{\boldsymbol{a}_{\mathbf{1}}}^{\top}\left(s+\boldsymbol{J}_{\boldsymbol{a}_{3}} \boldsymbol{a}_{3}+\boldsymbol{J}_{\boldsymbol{a}_{2}} \boldsymbol{a}_{2}\right) \\
& \boldsymbol{a}_{2}=-\left(\boldsymbol{J}_{\boldsymbol{a}_{2}}^{\top} \boldsymbol{J}_{\boldsymbol{a}_{2}}\right)^{-1} \boldsymbol{J}_{\boldsymbol{a}_{2}}^{\top}\left(s+\boldsymbol{J}_{\boldsymbol{a}_{3}} \boldsymbol{a}_{3}+\boldsymbol{J}_{\boldsymbol{a}_{1}} \boldsymbol{a}_{1}\right) \\
& \boldsymbol{a}_{3}=\left(\frac{\rho}{2} \mathbf{I}_{N}+\boldsymbol{J}_{\boldsymbol{a}_{3}}^{\top} \boldsymbol{J}_{\boldsymbol{a}_{3}}\right)^{-1}\left(\frac{\rho}{2} z-\frac{1}{2} \boldsymbol{y}-\boldsymbol{J}_{\boldsymbol{a}_{3}}^{\top}\left(s+\boldsymbol{J}_{\boldsymbol{a}_{2}} \boldsymbol{a}_{2}+\boldsymbol{J}_{\boldsymbol{a}_{1}} \boldsymbol{a}_{1}\right)\right)
\end{aligned}
$$

where $\mathbf{I}_{N}$ is the identity matrix of size $(N \times N)$ and

$$
\boldsymbol{J}_{\boldsymbol{a} \boldsymbol{\ell}}=\frac{\partial \boldsymbol{\psi}}{\partial \boldsymbol{a}_{\ell}^{\top}}=-\operatorname{diag}\left(e^{-\boldsymbol{b} \otimes \boldsymbol{d}_{\ell}}\right)\left(\mathbf{1}_{M} \otimes \mathbf{I}_{N}\right), \quad \forall \ell \in\{1,2,3\}
$$

and where $\operatorname{diag}($.$) is a vector-to-diagonal matrix transformation defined such that for a$ given vector $\boldsymbol{x}, \boldsymbol{X}=\operatorname{diag}(\boldsymbol{x})$ is a diagonal matrix whose diagonal is $\boldsymbol{x}$. More details regarding the derivation of equations (A.2), (A.3) and (A.4) are given in the subsequent subsection. The estimation of $\boldsymbol{d}_{\ell}, \forall \ell \in\{1,2,3\}$ is performed using the LM algorithm as follows:

$$
\Delta \boldsymbol{d}=\left(\boldsymbol{J}_{\boldsymbol{d}}^{\top} \boldsymbol{J}_{\boldsymbol{d}}+\mu \mathbf{I}_{3 N}\right)^{-1} \boldsymbol{J}_{\boldsymbol{d}}^{\top} \boldsymbol{\psi}
$$

where $\boldsymbol{d}=\left[\boldsymbol{d}_{1}^{\top}, \boldsymbol{d}_{2}^{\top}, \boldsymbol{d}_{3}^{\top}\right]^{\top}, \Delta \boldsymbol{d}$ denotes the difference between two estimates of $\boldsymbol{d}$ taken at two successive iterations, and the matrix $\boldsymbol{J}_{\boldsymbol{d}}$ of size $(M N \times 3 N)$ is obtained by concatenating $\boldsymbol{J}_{\boldsymbol{d}_{1}}, \boldsymbol{J}_{\boldsymbol{d}_{2}}$ and $\boldsymbol{J}_{\boldsymbol{d}_{3}}$ as follows: $\boldsymbol{J}_{\boldsymbol{d}}=\left[\boldsymbol{J}_{\boldsymbol{d}_{1}}, \boldsymbol{J}_{\boldsymbol{d}_{2}}, \boldsymbol{J}_{\boldsymbol{d}_{3}}\right]$ with $\boldsymbol{J}_{\boldsymbol{d}_{\boldsymbol{\ell}}}=\frac{\partial \boldsymbol{\psi}}{\partial \boldsymbol{d}_{\ell}^{\top}}$. Regarding the damping coefficient $\mu$, it is updated following the scheme proposed in (Madsen et al., 2004) with an initial guess being taken as the maximum element in the matrix $\boldsymbol{J}_{\boldsymbol{d}}^{\top} \boldsymbol{J}_{\boldsymbol{d}}$. As far as the latent variable $\boldsymbol{z}$ is concerned, it is computed as follows: (Boyd et al., 2011):

$$
z=\operatorname{prox}_{\phi, \frac{\lambda}{\rho}}\left(\boldsymbol{a}_{3}+\boldsymbol{y}\right)
$$


where the prox function stands for the proximity operator dealing with the non-smooth function $\phi$ (here $\left.\phi()=.\|.\|_{1}\right)$ initially proposed in (Ding, 2009). Regarding the Lagrangian variable $\boldsymbol{y}$, the dual ascent method is used such that (Boyd et al., 2011):

$$
\Delta y=a_{3}-z
$$

\section{A.1. Derivation of $\boldsymbol{a}_{1}$ (A.2), $\boldsymbol{a}_{2}$ (A.3) and $\boldsymbol{a}_{3}$ (A.4)}

Based on Kronecker product's properties (see Coloigner et al. (2014)) and equation (A.5), we have:

$$
\left(\mathbf{1}_{M} \otimes \boldsymbol{a}_{\ell}\right) \boxminus e^{-\boldsymbol{b} \otimes \boldsymbol{d}_{\ell}}=\operatorname{diag}\left(e^{-\boldsymbol{b} \otimes \boldsymbol{d}_{\ell}}\right)\left(\mathbf{1}_{M} \otimes \mathbf{I}_{N}\right) \boldsymbol{a}_{\ell}=-\boldsymbol{J}_{\boldsymbol{a}_{\ell}} \boldsymbol{a}_{\ell}
$$

then the all-voxel tri-exponential model (3) becomes:

$$
\boldsymbol{s}=\sum_{\ell=1}^{L=3}\left(\mathbf{1}_{M} \otimes \boldsymbol{a}_{\ell}\right) \square e^{-\boldsymbol{b} \otimes \boldsymbol{d}_{\ell}}+\boldsymbol{\epsilon}=-\sum_{\ell=1}^{L=3} \boldsymbol{J}_{\boldsymbol{a}_{\ell}} \boldsymbol{a}_{\ell}+\boldsymbol{\epsilon}
$$

Consequently, we can write $\psi=s+\boldsymbol{J}_{\boldsymbol{a}_{1}} \boldsymbol{a}_{1}+\boldsymbol{J}_{\boldsymbol{a}_{2}} \boldsymbol{a}_{2}+\boldsymbol{J}_{\boldsymbol{a}_{3}} \boldsymbol{a}_{3}$. Parameters $\boldsymbol{a}_{\ell}, \forall \ell \in$ $\{1,2,3\}$ are then computed as the solution of $\frac{\partial \mathcal{L}}{\partial \boldsymbol{a}_{\ell}^{\top}}=\mathbf{0}_{N}^{\top}, \forall \ell \in\{1,2,3\}$ with:

$$
\begin{aligned}
\mathcal{L} & =\|\boldsymbol{\psi}\|_{2}^{2}+\lambda\|z\|_{1}+\boldsymbol{y}^{\top}\left(\boldsymbol{a}_{3}-z\right)+\frac{\rho}{2}\left\|\boldsymbol{a}_{3}-z\right\|_{2}^{2} \\
& =\boldsymbol{\psi}^{\top} \boldsymbol{\psi}+\lambda\|z\|_{1}+\boldsymbol{y}^{\top}\left(\boldsymbol{a}_{3}-\boldsymbol{z}\right)+\frac{\rho}{2}\left(\boldsymbol{a}_{3}-\boldsymbol{z}\right)^{\top}\left(\boldsymbol{a}_{3}-\boldsymbol{z}\right)
\end{aligned}
$$

where $\mathbf{0}_{N}$ is an $N$-dimensional vector of zeros. Then we have:

$$
\begin{aligned}
\frac{\partial \mathcal{L}}{\partial \boldsymbol{a}_{\ell}^{\top}} & =\frac{\partial \mathcal{L}}{\partial \boldsymbol{\psi}^{\top}} \times \frac{\partial \boldsymbol{\psi}}{\partial \boldsymbol{a}_{\ell}^{\top}}=2 \boldsymbol{\psi}^{\top} \frac{\partial \boldsymbol{\psi}}{\partial \boldsymbol{a}_{\ell}^{\top}}, \forall \ell \in\{1,2\} \\
& =2\left(\boldsymbol{s}+\boldsymbol{J}_{\boldsymbol{a}_{1}} \boldsymbol{a}_{1}+\boldsymbol{J}_{\boldsymbol{a}_{2}} \boldsymbol{a}_{2}+\boldsymbol{J}_{\boldsymbol{a}_{3}} \boldsymbol{a}_{3}\right)^{\top} \boldsymbol{J}_{\boldsymbol{a}_{\ell}} \\
\frac{\partial \mathcal{L}}{\partial \boldsymbol{a}_{3}{ }^{\top}} & =2 \boldsymbol{\psi}^{\top} \frac{\partial \boldsymbol{\psi}}{\partial \boldsymbol{a}_{3}^{\top}}+\boldsymbol{y}^{\top}+\rho\left(\boldsymbol{a}_{3}-\boldsymbol{z}\right)^{\top}
\end{aligned}
$$

which immediately leads to:

$$
\begin{array}{r}
\boldsymbol{a}_{1}=-\left(\boldsymbol{J}_{\boldsymbol{a}_{1}}^{\top} \boldsymbol{J}_{\boldsymbol{a}_{1}}\right)^{-1} \boldsymbol{J}_{\boldsymbol{a}_{1}}^{\top}\left(s+\boldsymbol{J}_{\boldsymbol{a}_{3}} \boldsymbol{a}_{3}+\boldsymbol{J}_{\boldsymbol{a}_{2}} \boldsymbol{a}_{2}\right) \\
\boldsymbol{a}_{2}=-\left(\boldsymbol{J}_{\boldsymbol{a}_{2}}^{\top} \boldsymbol{J}_{\boldsymbol{a}_{2}}\right)^{-1} \boldsymbol{J}_{\boldsymbol{a}_{2}}^{\top}\left(s+\boldsymbol{J}_{\boldsymbol{a}_{3}} \boldsymbol{a}_{3}+\boldsymbol{J}_{\boldsymbol{a}_{1}} \boldsymbol{a}_{1}\right) \\
\boldsymbol{a}_{3}=\left(\frac{\rho}{2} \mathbf{I}_{N}+\boldsymbol{J}_{\boldsymbol{a}_{3}}^{\top} \boldsymbol{J}_{\boldsymbol{a}_{3}}\right)^{-1}\left[\frac{\rho}{2} z-\frac{1}{2} \boldsymbol{y}\right. \\
\left.\quad-\boldsymbol{J}_{\boldsymbol{a}_{3}}^{\top}\left(s+\boldsymbol{J}_{\boldsymbol{a}_{1}} \boldsymbol{a}_{1}+\boldsymbol{J}_{\boldsymbol{a}_{2}} \boldsymbol{a}_{2}\right)\right]
\end{array}
$$




\section{B. Technical materials on the solution of the $\mathbf{P 4}$ problem (7)}

As also discussed previously, the ADMM method (Boyd et al., 2011) is used to solve the $\mathrm{P} 4$ optimization problem (7). Thus, $\mathrm{P} 4$ (7) is reformulated as a minimization of its associated augmented Lagrangian function $\mathcal{L}_{1}$ leading to the P5 problem (8) recalled hereafter:

$$
P 5: \mathcal{L}_{1}=\|\boldsymbol{\psi}\|_{2}^{2}+\lambda\|\tilde{\boldsymbol{z}}\|_{1}+\tilde{\boldsymbol{y}}^{\top}\left(\tilde{\boldsymbol{a}_{3}}-\tilde{\boldsymbol{z}}\right)+\frac{\rho}{2}\left\|\tilde{\boldsymbol{a}_{3}}-\tilde{\boldsymbol{z}}\right\|_{2}^{2}
$$

Then, estimating parameters $\tilde{\boldsymbol{a}_{1}}, \tilde{\boldsymbol{a}_{2}}$ and $\tilde{\boldsymbol{a}_{3}}$ is performed by solving $\frac{\partial \mathcal{L}_{1}}{\partial \tilde{\boldsymbol{a}}_{1}^{\top}}=\mathbf{0}_{N}^{\top}$, ${ }_{580} \frac{\partial \mathcal{L}_{1}}{\partial \tilde{\boldsymbol{a}}_{2}{ }^{\top}}=\mathbf{0}_{N}^{\top}$ and $\frac{\partial \mathcal{L}_{1}}{\partial \tilde{\boldsymbol{a}}_{3}{ }^{\top}}=\mathbf{0}_{N}^{\top}$, respectively. Then the update rules of $\tilde{a}_{1}$ and $\tilde{\boldsymbol{a}}_{2}$ are given by:

$$
\begin{aligned}
& \tilde{\boldsymbol{a}_{1}}=-2\left(\boldsymbol{J}_{\tilde{\boldsymbol{a}_{1}}}^{\top} \boldsymbol{J}_{\tilde{\boldsymbol{a}_{1}}}\right)^{-1} \boldsymbol{J}_{\tilde{\boldsymbol{a}_{1}}}^{\top}\left(s+J_{a_{3}} \boldsymbol{a}_{3}+J_{\boldsymbol{a}_{2}} \boldsymbol{a}_{2}\right) \\
& \tilde{\boldsymbol{a}_{2}}=-2\left(\boldsymbol{J}_{\tilde{\boldsymbol{a}_{2}}}^{\top} \boldsymbol{J}_{\tilde{\boldsymbol{a}_{2}}}\right)^{-1} \boldsymbol{J}_{\tilde{\boldsymbol{a}_{2}}}^{\top}\left(s+\boldsymbol{J}_{\boldsymbol{a}_{3}} \boldsymbol{a}_{3}+\boldsymbol{J}_{\boldsymbol{a}_{\mathbf{1}}} \boldsymbol{a}_{1}\right)
\end{aligned}
$$

while the one for $\tilde{\boldsymbol{a}_{3}}$ is computed by rooting the following equation:

$$
4 \boldsymbol{\beta} \square\left(\tilde{\boldsymbol{a}}_{3}^{\square 3}\right)+\left(\rho \cdot \mathbf{1}_{N}+4 \boldsymbol{J}_{\boldsymbol{a}_{3}}^{\top} \boldsymbol{v}\right) \square \tilde{\boldsymbol{a}_{3}}+(\tilde{\boldsymbol{y}}-\rho \tilde{\boldsymbol{z}})=\mathbf{0}_{N}
$$

where $\boldsymbol{v}=\boldsymbol{s}-\left(\mathbf{1}_{M} \otimes \boldsymbol{a}_{1}\right) e^{-\boldsymbol{b} \otimes \boldsymbol{d}_{1}}-\left(\mathbf{1}_{M} \otimes \boldsymbol{a}_{2}\right) e^{-\boldsymbol{b} \otimes \boldsymbol{d}_{2}}, \boldsymbol{\beta}=\operatorname{diag}^{-1}\left(\boldsymbol{J}_{\boldsymbol{a}_{3}}^{\top} \boldsymbol{J}_{\boldsymbol{a}_{3}}\right)$ and where the Jacobian matrix $\boldsymbol{J}_{\tilde{\boldsymbol{a}_{\ell}}}$ is computed as follows (Coloigner et al., 2014, Lemme 2):

$$
\boldsymbol{J}_{\boldsymbol{a}_{\ell}}=\frac{\partial \boldsymbol{\psi}}{\partial \tilde{\boldsymbol{a}}_{\ell}^{\top}}=\frac{\partial \boldsymbol{\psi}}{\partial \boldsymbol{a}_{\ell}^{\top}} \times \frac{\partial \boldsymbol{a}_{\ell}}{\partial \tilde{\boldsymbol{a}}_{\ell}^{\top}}=\boldsymbol{J}_{\boldsymbol{a}_{\ell}} \times 2 \operatorname{diag}\left(\tilde{\boldsymbol{a}}_{\ell}\right)
$$

${ }_{585}$ A detailed derivation of equations (B.2), (B.3) and (B.4) is given in the subsequent subsection. As far as the estimation of parameters $\tilde{\boldsymbol{d}}_{\ell}, \forall \ell \in\{1,2,3\}$ is concerned, the LM method is employed. Then we have:

$$
\Delta \tilde{\boldsymbol{d}}=\left(\boldsymbol{J}_{\tilde{\boldsymbol{d}}}^{\top} \boldsymbol{J}_{\tilde{\boldsymbol{d}}}+\mu \mathbf{I}_{3 N}\right)^{-1} \boldsymbol{J}_{\tilde{\boldsymbol{d}}}^{\top} \boldsymbol{\psi}
$$

where $\tilde{\boldsymbol{d}}=\left[\tilde{\boldsymbol{d}}_{1}^{\top}, \tilde{\boldsymbol{d}}_{2}{ }^{\top}, \tilde{\boldsymbol{d}}_{3}{ }^{\top}\right]^{\top}$ and the Jacobian matrix $\boldsymbol{J}_{\tilde{\boldsymbol{d}}}$ is given by:

$$
\boldsymbol{J}_{\tilde{\boldsymbol{d}}}=\frac{\partial \boldsymbol{\psi}}{\partial \tilde{\boldsymbol{d}}^{\top}}=\frac{\partial \boldsymbol{\psi}}{\partial \boldsymbol{d}^{\top}} \times \frac{\partial \boldsymbol{d}}{\partial \tilde{\boldsymbol{d}}^{\top}}=\boldsymbol{J}_{\boldsymbol{d}} \times 2 \operatorname{diag}(\tilde{\boldsymbol{d}})
$$

As mentioned in Appendix A.1, the damping factor $\mu$ is computed as suggested in 590 (Madsen et al., 2004) but with an initial guess corresponding to the maximum entry 
of $\boldsymbol{J}_{\tilde{\boldsymbol{d}}}^{\top} \boldsymbol{J}_{\tilde{\boldsymbol{d}}}$. Similarly to equations (A.7) and (A.8), variables $\tilde{\boldsymbol{z}}$ and $\tilde{\boldsymbol{y}}$, are updated as follows:

$$
\begin{aligned}
\tilde{z} & =\operatorname{prox}_{\phi, \frac{\lambda}{\rho}}\left(\tilde{\boldsymbol{a}_{3}}+\tilde{\boldsymbol{y}}\right) \\
\Delta \tilde{\boldsymbol{y}} & =\tilde{\boldsymbol{a}_{3}}-\tilde{z}
\end{aligned}
$$

B.1. Derivation of $\tilde{\boldsymbol{a}_{1}}$ (B.2), $\tilde{\boldsymbol{a}_{2}}$ (B.3) and $\tilde{\boldsymbol{a}_{3}}$ (B.4)

Following the same gradient computation scheme adopted in Appendix A.1, we 595 have:

$$
\begin{aligned}
& \frac{\partial \mathcal{L}_{1}}{\partial \tilde{\boldsymbol{a}}_{\ell}^{\top}}=2 \boldsymbol{\psi}^{\top} \frac{\partial \boldsymbol{\psi}}{\partial \tilde{\boldsymbol{a}_{\ell}}}=2\left(s+\sum_{k=1}^{3} \boldsymbol{J}_{\boldsymbol{a}_{\boldsymbol{k}}} \boldsymbol{a}_{k}\right)^{\top} \boldsymbol{J}_{\tilde{\boldsymbol{a}_{\boldsymbol{\ell}}}}, \forall \ell \in\{1,2\} \\
& \frac{\partial \mathcal{L}_{1}}{\partial \tilde{\boldsymbol{a}}_{3}^{\top}}=2 \boldsymbol{\psi}^{\top} \frac{\partial \boldsymbol{\psi}}{\partial \tilde{\boldsymbol{a}}_{3}^{\top}}+\tilde{\boldsymbol{y}}^{\top}+\rho\left(\tilde{\boldsymbol{a}_{3}}-\tilde{\boldsymbol{z}}\right)^{\top}
\end{aligned}
$$

then immediately we obtain:

$$
\begin{aligned}
& \tilde{\boldsymbol{a}_{1}}=-2\left(\boldsymbol{J}_{\tilde{\boldsymbol{a}_{1}}}^{\top} \boldsymbol{J}_{\tilde{\boldsymbol{a}_{1}}}\right)^{-1} \boldsymbol{J}_{\tilde{a_{1}}}^{\top}\left(s+\boldsymbol{J}_{\boldsymbol{a}_{3}} \boldsymbol{a}_{3}+\boldsymbol{J}_{\boldsymbol{a}_{2}} \boldsymbol{a}_{2}\right) \\
& \tilde{\boldsymbol{a}_{2}}=-2\left(\boldsymbol{J}_{\tilde{\boldsymbol{a}_{2}}}^{\top} \boldsymbol{J}_{\tilde{\boldsymbol{a}_{2}}}\right)^{-1} \boldsymbol{J}_{\tilde{\boldsymbol{a}_{2}}}^{\top}\left(s+\boldsymbol{J}_{\boldsymbol{a}_{3}} \boldsymbol{a}_{3}+\boldsymbol{J}_{\boldsymbol{a}_{1}} \boldsymbol{a}_{1}\right)
\end{aligned}
$$

Regarding $\tilde{\boldsymbol{a}_{3}}$ we can write $\boldsymbol{J}_{\tilde{\boldsymbol{a}_{3}}}=\frac{\partial \boldsymbol{\psi}}{\partial \tilde{\boldsymbol{a}}_{3}}=\boldsymbol{J}_{\boldsymbol{a}_{3}} \cdot 2 \operatorname{diag}\left(\tilde{\boldsymbol{a}_{3}}\right)$. Also, we have $\boldsymbol{a}_{3}=\tilde{\boldsymbol{a}}^{\text {घ2 }}$, and $\psi=s+\sum_{\ell=1}^{L=3} J_{a_{\ell}} a_{\ell}$. Then we can write:

$$
\begin{aligned}
\frac{\partial \mathcal{L}_{1}}{\partial \tilde{\boldsymbol{a}}_{3}^{\top}} & =2\left(\boldsymbol{s}+\sum_{\ell=1}^{L=3} \boldsymbol{J}_{\boldsymbol{a}_{\boldsymbol{\ell}}} \boldsymbol{a}_{\ell}\right)^{\top} \boldsymbol{J}_{\tilde{\boldsymbol{a}_{3}}}+\tilde{\boldsymbol{y}}^{\top}+\rho\left(\tilde{\boldsymbol{a}_{3}}-\tilde{\boldsymbol{z}}\right)^{\top} \\
& =4 \boldsymbol{a}_{3}^{\top} \boldsymbol{J}_{\boldsymbol{a}_{3}}^{\top} \boldsymbol{J}_{\boldsymbol{a}_{3}} \operatorname{diag}\left(\tilde{\boldsymbol{a}_{3}}\right)+(\tilde{\boldsymbol{y}}-\rho \tilde{\boldsymbol{z}})^{\top}+\rho \tilde{\boldsymbol{a}}^{\top}+4 \boldsymbol{v}^{\top} \boldsymbol{J}_{\boldsymbol{a}_{3}} \operatorname{diag}\left(\tilde{\boldsymbol{a}_{3}}\right)
\end{aligned}
$$

where $v=s+J_{\boldsymbol{a}_{1}} \boldsymbol{a}_{1}+\boldsymbol{J}_{\boldsymbol{a}_{2}} \boldsymbol{a}_{2}$. Based on properties of the Hadamard product, we can write $4 \boldsymbol{v}^{\top} \boldsymbol{J}_{\boldsymbol{a}_{3}} \operatorname{diag}\left(\tilde{\boldsymbol{a}_{3}}\right)=4 \boldsymbol{v}^{\top} \boldsymbol{J}_{\boldsymbol{a}_{3}} \square \tilde{\boldsymbol{a}}^{\top}$. Also, according to equation (A.5), we have:

$$
\begin{aligned}
\boldsymbol{J}_{\boldsymbol{a}_{3}}^{\top} \boldsymbol{J}_{\boldsymbol{a}_{3}} & =\left(\mathbf{1}_{M} \otimes \mathbf{I}_{N}\right)^{\top} \operatorname{diag}\left(e^{-2 \boldsymbol{b} \otimes \boldsymbol{d}_{3}}\right)\left(\mathbf{1}_{M} \otimes \mathbf{I}_{N}\right) \\
& =\operatorname{diag}\left(\left[\sum_{m=1}^{M} e^{-2 b_{m} d_{3}^{(1)}}, \cdots, \sum_{m=1}^{M} e^{-2 b_{m} d_{3}^{(N)}}\right]\right)=\operatorname{diag}(\boldsymbol{\beta})
\end{aligned}
$$

which means that $\boldsymbol{J}_{\boldsymbol{a}_{3}}^{\top} \boldsymbol{J}_{\boldsymbol{a}_{3}}$ is a $(N \times N)$ diagonal matrix. In addition, based again on the Hadamard product's properties, the first term on the right-hand side of equation (B.14) 
can be written as:

$$
\begin{aligned}
& 4 \boldsymbol{a}_{3}^{\top} \boldsymbol{J}_{\boldsymbol{a}_{3}}^{\top} \boldsymbol{J}_{\boldsymbol{a}_{3}} \operatorname{diag}\left(\tilde{\boldsymbol{a}_{3}}\right)=4 \boldsymbol{a}_{3}^{\top} \cdot \operatorname{diag}(\boldsymbol{\beta}) \cdot \operatorname{diag}\left(\tilde{\boldsymbol{a}_{3}}\right) \\
& =4{\tilde{\boldsymbol{a}_{3}}}^{\top} \boxminus{\tilde{\boldsymbol{a}_{3}}}^{\top} \square \boldsymbol{\beta}^{\top} \square{\tilde{\boldsymbol{a}_{3}}}^{\top}=4 \boldsymbol{\beta}^{\top} \square\left(\tilde{\boldsymbol{a}}_{3}^{\varpi 3}\right)^{\top}
\end{aligned}
$$

Also, we have $\rho \tilde{\boldsymbol{a}}^{\top}=\rho \cdot \mathbf{1}_{N}^{\top} \square \tilde{\boldsymbol{a}}_{3}^{\top}$. Then, by substituting the latter expression together with (B.16) and the expression of $4 \boldsymbol{v}^{\top} \boldsymbol{J}_{\boldsymbol{a}_{3}} \operatorname{diag}\left(\tilde{\boldsymbol{a}_{3}}\right)$ in equation (B.15), we obtain:

$$
\frac{\partial \mathcal{L}_{1}}{\partial{\tilde{\boldsymbol{a}_{3}}}^{\top}}=4 \boldsymbol{\beta} \boxminus\left(\tilde{\boldsymbol{a}}^{\square 3}\right)+\left(\rho \cdot \mathbf{1}_{N}+4 \boldsymbol{J}_{\boldsymbol{a}_{3}}^{\top} \boldsymbol{v}\right) \boxminus \tilde{\boldsymbol{a}_{3}}+(\tilde{\boldsymbol{y}}-\rho \tilde{\boldsymbol{z}})
$$

which means that $\tilde{\boldsymbol{a}_{3}}$ can be found by rooting the above equation.

\section{Conflict of interest statement}

The authors declare that they do not have any conflict of interest that could inap-

\section{References}

Andreou, A., Koh, D., Collins, D., Blackledge, M., Wallace, T., Leach, M., Orton, M., 2013. Measurement reproducibility of perfusion fraction and pseudodiffusion coefficient derived by intravoxel incoherent motion diffusion-weighted MR imaging in normal liver and metastases. European radiology 23, 428-434.

Barbieri, S., Donati, O.F., Froehlich, J.M., Thoeny, H.C., 2016. Impact of the calculation algorithm on biexponential fitting of diffusion-weighted mri in upper abdominal organs. Magnetic resonance in medicine 75, 2175-2184.

Becker, H., Albera, L., Comon, P., Nunes, J., Gribonval, R., Fleureau, J., Guillotel, P., Merlet, I., 2017. SISSY: an efficient and automatic algorithm for the analysis of EEG sources based on structured sparsity. NeuroImage 157, 157172. URL: https://doi .org/10.1016/j .neuroimage .2017 .05 .046 , doi:10. 1016/j . neuroimage . 2017.05 .046 . 
Boyd, S., Parikh, N., Chu, E., Peleato, B., Eckstein, J., 2011. Distributed optimization and statistical learning via the alternating direction method of multipliers. Foundations and Trends in Machine Learning 3, 1-122.

Boyd, S., Vandenberghe, L., 2004. Convex optimization. Cambridge University Press. Cercueil, J., Petit, J., Nougaret, S., Soyer, P., Fohlen, A., Pierredon-Foulongne, M., Schembri, V., Delhom, E., Schmidt, S., Denys, A., et al., 2015. Intravoxel incoherent motion diffusion-weighted imaging in the liver: comparison of mono-, bi-and triexponential modelling at 3.0-T. European radiology 25, 1541.

Coloigner, J., Karfoul, A., Albera, L., Comon, P., 2014. Line search and trust region strategies for canonical decomposition of semi-nonnegative semi-symmetric 3rd order tensors. Linear Algebra and Applications, Elsevier-Academic Press 450, 334-374.

Ding, L., 2009. Reconstructing cortical current density by exploring sparseness in the transform domain. Physics in Medicine and Biology 54, 2683 - 2697.

Dyvorne, H., Galea, N., Nevers, T., Fiel, M., Carpenter, D., Wong, E., Orton, M., de Oliveira T. Feiweier, A., Vachon, M., Babb, J., Taouli, B., 2013. Diffusionweighted imaging of the liver with multiple b values: effect of diffusion gradient polarity and breathing acquisition on image quality and intravoxel incoherent motion parameters--a pilot study. Radiology 266, 902-909.

Gambarota, G., Hitti, E., Leporq, B., Saint-Jalmes, H., Beuf, O., 2017. Eliminating the blood-flow confounding effect in intravoxel incoherent motion (IVIM) using the non-negative least square analysis in liver. Magnetic resonance in medicine 77, $310-317$.

Gudbjartsson, H., Patz, S., 2008. The Rician Distribution of Noisy MRI data. Magnetic Resonance in Medicine 34, 910-914.

Guiu, B., Petit, J., Capitan, V., Aho, S., Masson, D., Lefevre, P., Favelier, S., Loffroy, R., Vergès, B., Hillon, P., et al., 2012. Intravoxel incoherent motion diffusion-weighted imaging in nonalcoholic fatty liver disease: a 3.0-t mr study. Radiology 265, 96-103. 
Hayashi, T., Miyati, T., Takahashi, J., Fukuzawa, K., Sakai, H., Tano, M., Saitoh, S., 2013. Diffusion analysis with triexponential function in liver cirrhosis. Journal of Magnetic Resonance Imaging 38, 148-153.

Komodakis, N., Pesquet, J., 2015. Playing with Duality: An overview of recent primaldual approaches for solving large-scale optimization problems. IEEE Signal Processing Magazine URL: https://hal . archives-ouvertes . fr/hal-01010437.

Lawson, C., Hanson, R., 1995. Solving least squares problems. volume 15. Siam.

Le Bihan, D., 2008. Intravoxel incoherent motion perfusion MR imaging: a wake-up call. Radiology 249, 748-752.

Le Bihan, D., Breton, E., Lallemand, D., Aubin, M., Vignaud, J., Laval-Jeantet, M., 1988. Separation of diffusion and perfusion in intravoxel incoherent motion MR imaging. Radiology 168, 497-505.

Le Bihan, D., Breton, E., Lallemand, D., Grenier, P., Cabanis, E., Laval-Jeantet, M., 1986. MR imaging of intravoxel incoherent motions: application to diffusion and perfusion in neurologic disorders. Radiology 161, 401-407.

Leporq, B., Saint-Jalmes, H., Rabrait, C., Pilleul, F., Guillaud, O., Dumortier, J., Scoazec, J., Beuf, O., 2015. Optimization of intra-voxel incoherent motion imaging at 3.0 tesla for fast liver examination. Journal of Magnetic Resonance Imaging 41, $1209-1217$

Lipton, M.L., 2008. Totally accessible MRI: A user's guide to principles technology and applications. Springer, New York.

Liu, J., Gambarota, G., Shu, H., Jiang, L., Leporq, B., Beuf, O., Karfoul, A., 2017. Efficient sparsity-based algorithm for parameter estimation of the tri-exponential intra voxel incoherent motion (IVIM) model: Application to diffusion-weighted MR imaging in the liver, in: Computational Advances in Multi-Sensor Adaptive Processing (CAMSAP), 2017 IEEE 7th International Workshop, Curacao, Netherlands Antilles. 
Liu, J., Gambarota, G., Shu, H., Jiang, L., Leporq, B., Beuf, O., Karfoul, A., 2018. All-in-one approach for constrained all-voxel tri-exponential IVIM model identification: Application to diffusion weighted MR imaging in the liver, in: To appear in the proceedings of IEEE Asilomar Conference on Signal, Systems and Computers, Asilomar Hotel and Conference Grounds, Pacific Grove, CA, USA.

685 Loomba, R., Sanyal, A., 2013. The global NAFLD epidemic. Nature Reviews Gastroenterology and Hepatology 10, 686.

Madsen, K., Nielsen, H., Tingleff, O., 2004. Methods for non-linear least squares problems. Informatics and Mathematical Modeling, Technical University of Denmark URL: Availableonline: http://www.imm.dtu, dk.

Nocedal, J., Wright, S., 2006. Numerical Optimization. 2 ed., Springer, New York.

Pasquinelli, F., Belli, G., Mazzoni, L., Grazioli, L., Colagrande, S., 2011. Magnetic resonance diffusion-weighted imaging: quantitative evaluation of age-related changes in healthy liver parenchyma. Magnetic Resonance Imaging 29, 805-812. 